\title{
MARTINGALES ON JUMP PROCESSES. II: APPLICATIONS*
}

\author{
R. BOEL, P. VARAIYA AND E. WONG $\dagger$
}

1. Introduction and summary. This paper is concerned with applying the theory of martingales of jump processes to various problems arising in communication and control. It parallels the approaches which have been recently discovered in dealing with similar problems where the underlying stochastic process is Brownian motion. Indeed these approaches have recently been extended, starting with the work of Snyder [14], [16], [30] and Brémaud [6], [28], to the case of the Poisson process and its transformations. The paper can then be regarded as a sweeping generalization to this recent work.

The paper can also be considered as an illustration of an abstract view and a set of instructions which must be followed to obtain certain concrete results in the areas of communication and control. It is hoped that this tutorial function will also be served.

Two results from the abstract theory of martingales form the basis of this abstract view. The first consists of the differentiation rule and the associated stochastic calculus for martingales and semi-martingales [1], and its application to the so-called "exponentiation" formula [2]. The second result consists of the earlier Doob-Meyer decomposition theorem for supermartingales [3]. In order to follow the abstract view, one also needs a third set of results, the so-called "martingale representation" theorems for specific processes. These results form a bridge between the abstract theory and the concrete applications. The representation results used here have been obtained in [4], hence the paper can also be viewed as a continuation of that work.

The paper is organized in the following manner. In the next section are presented many definitions, notations and results from [1], [2], [3], [4] which will be used in the succeeding development. These preliminaries are certainly longer than can be considered proper, and are justified partly to serve the tutorial function, partly because there is no consensus of usage in the literature, and lastly because some of the published literature contains errors and inaccurate or misleading statements which can be exposed only within a carefully and completely developed context.

Section 3 is concerned with showing the "global" existence of jump processes over a finite or infinite interval which satisfy certain local descriptions. Existence of such processes is obtained by transforming the laws of "known" processes by an absolutely continuous transformation. We also present a wide class of point processes which can be so transformed to yield solutions to prespecified local

* Received by the editors December 21, 1973, and in revised form August 13, 1974.

$\dagger$ Department of Electrical Engineering and Computer Sciences and the Electronics Research Laboratory, University of California, Berkeley, California 94720. This research was supported by the National Science Foundation under Grant GK-10656X3 and the Army Research Office-Durham under Contract DAHC04-67-C-0046. The work of the first author was also supported by an ESRONASA International Fellowship. 
descriptions. Sufficient conditions are derived which guarantee when this technique is applicable. The question of uniqueness of the solutions is settled for a wide class of local descriptions.

Section 4 deals with a specific problem in communication theory, namely the calculation of the likelihood ratio of a process which may be governed by one or two absolutely continuous probability laws. The techniques for $\S \S 3$ and 4 are the same. Section 5 is concerned with estimating certain random variables or processes which are statistically related to an observed process. The emphasis here is on obtaining "recursive" filters. As special cases one obtains a "closed form" solution for some of the situations where the estimated process is Markovian. Applications to optimal control will be made in a future paper.

Throughout, there has been an attempt to link up the results with those which have already appeared in the literature in as precise a manner as limitations of space permit. Any omissions are due to oversight of the authors.

2. Preliminaries and formulations. This section describes most of the results from the literature which are necessary to the sequel. Section 2.1 is definitional in nature. Sections $2.2-2.7$ are taken mainly from [1], $\$ 2.8$ is taken from [2], the remainder is from [4].

2.1. Processes. Throughout $\Omega$ is a fixed space, the sample space. The time interval of interest is $R_{+}=[0, \infty)$ unless specified otherwise. For each $t$ let $\mathscr{F}_{t}$ be a $\sigma$-field of subsets of $\Omega$. It will always be assumed that the family $\mathscr{F}_{t}, t \in R_{+}$, is increasing, i.e., $\mathscr{F}_{s} \subset \mathscr{F}_{t}$ for $s \leqq t$ and right-continuous, i.e., $\mathscr{F}_{t}=\bigcap_{s>t} \mathscr{F}_{s}$. Let $\mathscr{F}=\bigvee_{t} \mathscr{F}_{t}$ be the smallest $\sigma$-field containing all the $\mathscr{F}_{t}$. Let $P$ be a probability measure on $(\Omega, \mathscr{F})$. Thus one has a family of probability spaces $\left(\Omega, \mathscr{F}_{t}, P\right)$. It will always be assumed that probability spaces are complete.

Let $(Z, \mathscr{Z})$ be a measurable space. Let $x: \Omega \times R_{+} \rightarrow Z$ be a function such that $\left\{\omega \mid x_{t}(\omega) \in B\right\} \in \mathscr{F}_{t}$ for all $B \in \mathscr{Z}, t \in R_{+}$. Then $\left(x_{t}, \mathscr{F}_{t}, P\right)$ is a (stochastic) process. Thus every process has attached to it a family $\left(\Omega, \mathscr{F}_{t}, P\right), t \in R_{+}$, of probability spaces. The same function $x$ defines a different process if either the family $\mathscr{F}_{t}$ or the measure $P$ is changed. When the context makes it clear we write $\left(x_{t}, \mathscr{F}_{t}\right)$ or $\left(x_{t}, P\right)$ or $x_{t}$ instead of $\left(x_{t}, \mathscr{F}_{t}, P\right)$. If $\left(x_{t}, \mathscr{F}_{t}, P\right)$ is a process, then so is $\left(x_{t}, \mathscr{F}_{t}^{x}, P\right)$ where $\mathscr{F}_{t}^{x}$ is the sub- $\sigma$-field of $\mathscr{F}_{t}$ generated by $x_{s}, s \leqq t$, and $P$ is the restriction to $\mathscr{F}^{x}=\bigvee_{t} \mathscr{F}_{t}^{x}$. Two processes $\left(x_{t}, \mathscr{F}_{t}, P\right)$ and $\left(y_{t}, \mathscr{F}_{t}, P\right)$ are said to be modifications or versions of one another if $x_{t}=y_{t}$ a.s. $P$ for each $t$, the set $\left\{x_{t} \neq y_{t}\right\}$ may vary with $t$. They are said to be indistinguishable if there is a set $N$ with $P(N)=0$ such that for $\omega \notin N, x_{t}(\omega)=y_{t}(\omega)$ for all $t$. Given $(\Omega, \mathscr{F}, P)$, a random variable, or r.v., with values in $(Z, \mathscr{Z})$ is a $\mathscr{F}$-measurable map from $\Omega$ into $Z$. Unless explicitly stated otherwise all r.v.s and processes take values in $(R \cup\{\infty\}$, $\mathscr{B})$, where $\mathscr{B}$ is the Borel field.

2.2. Stopping times. Consider a family $\left(\Omega, \mathscr{F}_{t}, P\right)$. A nonnegative r.v. $T$ is a stopping time, s.t., of the family if

$$
\{T \leqq t\} \in \mathscr{F}_{t} \text { for all } t
$$

The s.t. $T$ is said to be predictable if there exists an increasing sequence of s.t.s, 
$S_{1} \leqq S_{2} \leqq \cdots$, such that

$$
P\left\{T=0 \text { or } S_{k}<T \text { for all } k \text { and } \lim _{k \rightarrow \infty} S_{k}=T\right\}=1
$$

The s.t. $T$ is said to be totally inaccessible if $T>0$ a.s. and if for every increasing sequence of s.t.s $S_{1} \leqq S_{2} \leqq \cdots$,

$$
P\left\{S_{k}<T \text { for all } k \text { and } \lim _{k \rightarrow \infty} S_{k}=T<\infty\right\}=0 .
$$

2.3. Martingales and increasing processes. A process $\left(m_{t}, \mathscr{F}_{t}, P\right)$ is said to be a (uniformly integrable) martingale if the collection $\left\{m_{t} \mid t \in R^{+}\right\}$of r.v.s is uniformly integrable, and if $E\left(m_{t} \mid \mathscr{F}_{s}\right)=m_{s}$ a.s. for $s \leqq t$. The collection of all such martingales, for which $m_{0}=0$, is denoted $\mathscr{M}^{1}=\mathscr{M}^{1}\left(\mathscr{F}_{t}, P\right) .\left(m_{t}, \mathscr{F}_{t}, P\right)$ is said to be a local martingale if there is an increasing sequence of s.t.s $S_{k}$, with $S_{k} \rightarrow \infty$ a.s. such that

$$
\left(m_{t \wedge S_{k}} I_{\left\{S_{k}>0\right\}}, \mathscr{F}_{t}, P\right) \in \mathscr{M}^{1} \text { for each } k
$$

The collection is denoted $\mathscr{M}_{\text {loc }}^{1}\left(\mathscr{F}_{t}, P\right) \cdot\left(m_{t}, \mathscr{F}_{t}, P\right)$ is a square integrable martingale if $m_{t} \in \mathscr{M}^{1}$ and if $\sup _{t} E m_{t}^{2}<\infty$. The collection is denoted $\mathscr{M}^{2}\left(\mathscr{F}_{t}, P\right)$ and the class of locally square integrable martingales $\mathscr{M}_{\text {loc }}^{2}\left(\mathscr{F}_{t}, P\right)$ is defined analogously. It is obvious that $\mathscr{M}_{\text {loc }}^{2} \subset \mathscr{M}_{\text {loc }}^{1}$.

Each $m_{t} \in \mathscr{M}_{\text {loc }}^{1}$ has a version whose sample paths are right-continuous and have left-hand limits. Clearly such a version is unique, i.e., unique modulo indistinguishability. It will always be assumed that local martingales have sample paths with this continuity property.

A process $\left(a_{t}, \mathscr{F}_{t}, P\right)$ is said to be increasing if $a_{0}=0$ a.s. and if its sample paths are right-continuous and nondecreasing. The collection is denoted

$$
\begin{gathered}
\mathscr{A}_{0}^{+}\left(\mathscr{F}_{t}, P\right) . \\
\mathscr{A}_{0}=\mathscr{A}_{0}^{+}-\mathscr{A}_{0}^{+}=\left\{a_{t}-a_{t}^{\prime} \mid a_{t} \in \mathscr{A}_{0}^{+}, a_{t}^{\prime} \in \mathscr{A}_{0}^{+}\right\} . \\
\mathscr{A}^{+}=\left\{a_{t} \in \mathscr{A}_{0}^{+} \mid \sup _{t} E a_{t}<\infty\right\}, \quad \mathscr{A}=\mathscr{A}^{+}-\mathscr{A}^{+} .
\end{gathered}
$$

Members of $\mathscr{A}^{+}(\mathscr{A})$ are said to be integrable (or have integrable variation). $a_{t} \in \mathscr{A}_{0}^{+}$is said to be locally integrable $a_{t} \in \mathscr{A}_{\text {loc }}^{+}$if there is an increasing sequence of s.t.s $S_{k} \rightarrow \infty$ a.s. such that $a_{t \wedge S_{k}} \in \mathscr{A}^{+}$for all $k . \mathscr{A}_{\text {loc }}=\mathscr{A}_{\text {loc }}^{+}-\mathscr{A}_{\text {loc }}^{+}$.

Semimartingales. A process $\left(s_{t}, \mathscr{F}_{t}, P\right)$ is a semimartingale, respectively local semimartingale, if it can be expressed as $s_{t}=s_{0}+m_{t}+a_{t}$, where $m_{t} \in \mathscr{M}^{1}\left(\mathscr{F}_{t}, P\right)$ and $a_{t} \in \mathscr{A}\left(\mathscr{F}_{t}, P\right)$, respectively, $m_{t} \in \mathscr{M}_{\text {loc }}^{1}\left(\mathscr{F}_{t}, P\right)$ and $a_{t} \in \mathscr{A}_{0}\left(\mathscr{F}_{t}, P\right)$. The families are respectively denoted $\mathscr{S}\left(\mathscr{F}_{t}, P\right)$ and $\mathscr{S}_{\text {loc }}\left(\mathscr{F}_{t}, P\right)$.

2.4. Predictable processes. The family of all processes $\left(y_{t}, \mathscr{F}_{t}, P\right)$ which have left-continuous sample paths generates a $\sigma$-field $\mathscr{P}=\mathscr{P}\left(\mathscr{F}_{t}\right) \subset \mathscr{F} \otimes \mathscr{B}$, where $\mathscr{B}$ is the Borel field of $R_{+}$, with respect to which the functions $(\omega, t) \mapsto y_{t}(\omega)$ are measurable. $\mathscr{P}$ is called the predictable $\sigma$-field, and every process $\left(y_{t}, \mathscr{F}_{t}, P\right)$ 
which is $\mathscr{P}$-measurable is called a predictable process. Note that if $\mathscr{F}_{t} \subset \mathscr{G}_{t}$, then $\mathscr{P}\left(\mathscr{F}_{t}\right) \subset \mathscr{P}\left(\mathscr{G}_{t}\right)$.

For $\left(a_{t}, \mathscr{F}_{y}, P\right) \in \mathscr{A}_{0}$,

$$
\begin{gathered}
L^{p}\left(a_{t}\right)=\left\{y_{t} \mid\left(y_{t}, \mathscr{F}_{t}, P\right) \text { is predictable and } E \int_{0}^{\infty}\left|y_{t}\right|^{p}\left|d a_{t}\right|<\infty\right\} . \\
L_{\text {loc }}^{p}\left(a_{t}\right)=\left\{y_{t} \mid \text { there is a sequence of s.t.s } S_{k} \rightarrow \infty\right. \text { such that } \\
\left.y_{t} I_{\left\{t \leqq S_{k}\right\}} \in L^{p}\left(a_{t}\right) \text { for each } k\right\} .
\end{gathered}
$$

The integrals above are Stieltjes integrals.

2.5. Quadratic variation. Two martingales $m_{t}, n_{t}$ in $\mathscr{M}_{\mathrm{loc}}^{1}$ are orthogonal if their product, $m_{t} n_{t} \in \mathscr{M}_{\mathrm{loc}}^{1} \cdot m_{t} \in \mathscr{M}_{\mathrm{loc}}^{1}$ is continuous if its sample paths are continuous; it is said to be discontinuous if it is orthogonal to every continuous martingale. Every $m_{t} \in \mathscr{M}_{\text {loc }}^{1}$ has a unique decomposition,

$$
m_{t}=m_{t}^{c}+m_{t}^{d}
$$

such that $m_{t}^{c}$ is continuous and $m_{t}^{d}$ is discontinuous. Clearly if $m_{t} \in \mathscr{M}_{\text {loc }}^{1}$ is continuous, then it is in $\mathscr{M}_{\text {loc }}^{2}$. To every path $m_{t}, n_{t}$ in $\mathscr{M}_{\text {loc }}^{2}$ is associated a unique predictable process, denoted $\langle m, n\rangle_{t}$ or $\left(\left\langle m_{t}, n_{t}\right\rangle, \mathscr{F}_{t}, P\right)$ such that $\langle m, n\rangle_{t} \in \mathscr{A}_{\text {loc }}^{+}$, and

$$
\left(m_{t} n_{t}-\langle m, n\rangle_{t}\right) \in \mathscr{M}_{\mathrm{loc}}^{1}\left(\mathscr{F}_{t}, P\right) .
$$

$\langle m, n\rangle_{t}$ is called the predictable quadratic covariation of $m_{t}, n_{t}$. For $m_{t} \in \mathscr{M}_{\mathrm{loc}}^{2}$, $\langle m\rangle_{t}=\langle m, m\rangle_{t}$ is the predictable quadratic variation of $m_{t}$. Note that generally $\langle m, n\rangle$ depends crucially upon the family $\left(\mathscr{F}_{t}, P\right)$.

If $m_{t}, n_{t}$ in $\mathscr{M}_{\text {loc }}^{1}$ have the decompositions $m_{t}=m_{t}^{c}+m_{t}^{d}, n_{t}=n_{t}^{c}+n_{t}^{d}$, then the process

$$
[m, n]_{t}=\left[m_{t}, n_{t}\right]=\left\langle m^{c}, n^{c}\right\rangle_{t}+\sum_{s \leqq t} \Delta m_{s}^{\prime} \Delta n_{s}
$$

where $\Delta m_{s}=m_{s}-m_{s^{-}}, \Delta n_{s}=n_{s}-n_{s^{-}}$, is called the quadratic covariation of $m_{t}$, $n_{t}$ and $\left[m_{t}\right]=\left[m_{t}, m_{t}\right]$ is the quadratic variation of $m_{t}$. It turns out that

$$
m_{t} n_{t}-[m, n]_{t} \in \mathscr{M}_{\mathrm{loc}}^{1}
$$

so that if, furthermore, $m_{t}, n_{t}$ are in $\mathscr{M}_{\text {loc }}^{2}$, then

$$
[m, n]_{t}-\langle m, n\rangle_{t} \in \mathscr{M}_{\mathrm{loc}}^{1}
$$

2.6. Stochastic integration. If $m_{t} \in \mathscr{M}_{\mathrm{loc}}^{2}\left(\mathscr{F}_{t}, P\right)$ and $\phi_{t} \in L_{\mathrm{loc}}^{2}\left(\langle m\rangle_{t}\right)$, then $\phi_{t} \in L_{\mathrm{loc}}^{1}\left(\langle m, n\rangle_{t}\right)$ for all $n_{t} \in \mathscr{M}_{\mathrm{loc}}^{2}\left(\mathscr{F}_{t}, P\right)$ and there is a unique process, denoted $(\phi \circ m)_{t} \in \mathscr{M}_{\text {loc }}^{2}\left(\mathscr{F}_{t}, P\right)$, which satisfies

$$
\langle\phi \circ m, n\rangle_{t}=\int_{0}^{t} \phi_{s} d\langle m, n\rangle_{s} \quad \text { for all } n_{t} \in \mathscr{M}_{\mathrm{loc}}^{2} .
$$

The integral on the right is a Stieltjes integral. If $m_{t} \notin \mathscr{M}_{\text {loc }}^{2}$ then one cannot define a stochastic integral in this way. Two other possibilities are open. 
If $m_{t}=m_{t}^{c}+m_{t}^{d} \in \mathscr{M}_{\mathrm{loc}}^{1}\left(\mathscr{F}_{t}, P\right)$, if $m_{t}^{d} \in \mathscr{A}_{\mathrm{loc}}\left(\mathscr{F}_{t}, P\right)^{1}$ and if $\phi_{t} \in L_{\mathrm{loc}}^{2}\left(\left\langle m^{c}\right\rangle_{t}\right)$ $\cap L_{\mathrm{loc}}^{1}\left(m_{t}^{d}\right)$, then the process

$$
(\phi \circ m)_{t}=\left(\phi \circ m^{c}\right)_{t}+\int_{0}^{t} \phi_{s} d m_{s}^{d} \in \mathscr{M}_{\mathrm{loc}}^{1}\left(\mathscr{F}_{t}, P\right),
$$

where $\left(\phi \circ m^{c}\right)_{t}$ is defined as in (2.1) whereas the second integral is a Stieltjes integral.

Finally if $m_{t} \in \mathscr{M}_{\mathrm{loc}}^{1}$ and if $\left(\phi_{t}, \mathscr{F}_{t}, P\right)$ is a locally bounded ${ }^{2}$ predictable process, then there exists a unique process $(\phi \circ m)_{t} \in \mathscr{M}_{\mathrm{loc}}^{1}$ which satisfies

$$
[\phi \circ m, n]_{t}=\int_{0}^{t} \phi_{s} d[m, n]_{s} \quad \text { for all } n \in \mathscr{M}_{\mathrm{loc}}^{1}
$$

The integral on the right is not in general a Stieltjes integral unless $[m, n]_{t} \in \mathscr{A}_{1 \mathrm{loc}}$. The precise interpretation of this integral is not given here since it is seldom used below. For details see [1].

The process $(\phi \circ m)_{t}$ is called the stochastic integral of $\phi$ with respect to $m$. Note that if $(\phi \circ m)$ makes sense according to more than one of the three possibilities (2.1), (2.2) or (2.3), then the resulting stochastic integrals coincide.

2.7. Differentiation formula. Let $s_{t}=s_{0}+m_{t}+a_{t} \in \mathscr{S}_{\text {loc }}\left(\mathscr{F}_{t}, P\right)$. The decomposition is not unique. If $s_{t}=s_{0}+m_{t}^{\prime}+a_{t}^{\prime}$ is another decomposition, then the continuous parts $m_{t}^{c}, m_{t}^{\prime c}$ of the local martingale are indistinguishable. This unique continuous local martingale is denoted $s_{t}^{c}$.

Let $s_{t}=\left(s_{t}^{1}, \cdots, s_{t}^{n}\right)$ be a process with values in $R^{n}$ such that $s_{t}^{i} \in \mathscr{S}_{\text {loc }}\left(\mathscr{F}_{t}, P\right)$, $i=1, \cdots, n$. Let $F: R^{n} \rightarrow R$ be a twice continuously differentiable function. Then the following differentiation formula holds:

$$
\begin{aligned}
F\left(s_{t}\right)= & F\left(s_{0}\right)+\int_{0}^{t} \sum_{i=1}^{n} \frac{\partial F}{\partial x_{i}}\left(s_{\tau^{-}}\right) d s_{\tau}^{i}+\frac{1}{2} \int_{0}^{t} \sum_{i, j=1}^{n} \frac{\partial^{2} F}{\partial x_{i} \partial x_{j}}\left(s_{\tau^{-}}\right) d\left\langle s^{i c}, s^{j c}\right\rangle_{\tau} \\
& +\sum_{\tau \leqq t}\left[F\left(s_{\tau}\right)-F\left(s_{\tau^{-}}\right)-\sum_{i=1}^{n} \frac{\partial F}{\partial x_{i}}\left(s_{\tau^{-}}\right)\left(s_{\tau}^{i}-s_{\tau^{-}}^{i}\right)\right] .
\end{aligned}
$$

As a special case one obtains the very useful "product" rule. Suppose $m_{t}$ and $n_{t}$ are in $\mathscr{M}_{\text {loc }}^{1}$. Then (since $m_{0}=n_{0}=0$ ), and recalling the definition of $[m, n]_{t}$,

$$
m_{t} n_{t}=\int_{0}^{t} m_{s^{-}} d n_{s}+\int_{0}^{t} n_{s^{-}} d m_{s}+[m, n]_{t} .
$$

2.8. The exponentiation formula. Let $s \in \mathscr{P}_{\text {loc }}\left(\mathscr{F}_{t}, P\right)$ with $s_{0}=0$. Then there is a unique process $y_{t} \in \mathscr{S}_{\text {loc }}\left(\mathscr{F}_{t}, P\right)$ which satisfies the equation

$$
y_{t}=y_{0}+\int_{0}^{t} y_{\tau}-d s_{\tau}, \quad t \geqq 0,
$$

\footnotetext{
${ }^{1}$ This is a nontrivial restriction on $m_{t}^{d}$. It holds for the discontinuous martingales to be introduced in $\S 2.9$ below.

${ }^{2} \phi_{t}$ is locally bounded if there is an increasing sequence of s.t.s $S_{k} \rightarrow \infty$ such that the process $\phi_{t \wedge S_{k}} I_{\left\{S_{k}>0\right\}}$ is bounded for all $k$. Note that if $\phi_{t}$ is a right-continuous process, having left-hand limits, then the process $\psi_{t}=\phi_{t-}$ is locally bounded.
} 
for a prespecified $\mathscr{F}_{0}$-measurable $y_{0}$, and $y_{t}$ is given explicitly by

$$
y_{t}=y_{0} \exp \left(s_{t}-\frac{1}{2}\left\langle s^{c}, s^{c}\right\rangle_{t}\right) \cdot \prod_{\tau \leqq t}\left(1+\Delta s_{\tau}\right) e^{-\Delta s} \tau,
$$

where the second term converges a.s. $y_{t}$ is called the exponential of $s_{t}$ and is sometimes denoted $y_{t}=\mathscr{E}\left(s_{t}\right)$. Evidently $\mathscr{E}\left(s_{t}\right) \geqq 0$ a.s. if $y_{0} \geqq 0$ a.s., and if $1+\Delta s \geqq 0$ a.s. If, in addition, $m_{0} \geqq 0$ and $m_{t}-m_{0} \in \mathscr{M}_{\text {loc }}^{1}\left(\mathscr{F}_{t}, P\right)$, then $\left(\mathscr{E}\left(m_{t}\right), \mathscr{F}_{t}, P\right)$ is a supermartingale, i.e.,

$$
E\left(\mathscr{E}\left(m_{t}\right) \mid \mathscr{F}_{s}\right) \leqq \mathscr{E}\left(m_{s}\right), \quad s \leqq t
$$

and so in particular,

$$
E\left(\mathscr{E}\left(m_{t}\right)\right) \leqq E\left(m_{0}\right), \quad t \geqq 0 .
$$

Finally if $m_{t} \in \mathscr{M}^{1}$ is bounded, then $\mathscr{E}\left(m_{t}\right)$ is a martingale.

2.9. The fundamental jump process. Let $\left(\Omega, \mathscr{F}_{t}, P\right)$ be a family of spaces and let $\left(x_{t}, \mathscr{F}_{t}, P\right)$ be a process with values in $(Z, \mathscr{Z})$ such that all the sample paths of $x$ are piecewise constant and have only a finite number of discontinuities in every finite interval, and such that the sample paths are right-continuous, i.e., for all $\omega$, $t$ there is $\varepsilon_{0}>0$ such that $x_{t}(\omega)=x_{t+\varepsilon}(\omega)$ for $0 \leqq \varepsilon \leqq \varepsilon_{0}$. Let $T_{n}, n=0,1, \cdots$, denote the jump times of the process, defined inductively by $T_{0} \equiv 0$ and

$$
T_{n+1}(\omega)=\left\{\begin{array}{l}
\inf \left\{t \mid t>T_{n}(\omega), x_{t}(\omega) \neq x_{T_{n}}(\omega)\right\}, \quad n \geqq 0, \\
\infty \text { if the set above is empty }
\end{array}\right.
$$

$\left(x_{t}, \mathscr{F}_{t}, P\right)$ is a fundamental jump process, or a fundamental process, f.p., with values in $(Z, \mathscr{Z})$, if in addition,

(i) $(Z, \mathscr{Z})$ is a Blackwell space, and then it turns out that the jump times are s.t.s, and

(ii) The s.t.s $T_{n}$ are totally inaccessible.

Evidently if $\left(x_{t}, \mathscr{F}_{t}, P\right)$ is a f.p., so is $\left(x_{t}, \mathscr{F}_{t}^{x}, P\right)$, where $\mathscr{F}_{t}^{x}$ is the sub- $\sigma$-field of $\mathscr{F}_{t}$ generated by $x_{s}, s \leqq t$. For each $B \in \mathscr{Z}$, let

$$
P(B, t)=\sum_{s \leqq t} I_{\left\{x_{s}^{-} \neq x_{s}\right\}} I_{\left\{x_{s} \in B\right\}}
$$

be the number of jumps of $x$ which occur prior to $t$ and which end in the set $B$.

Associated with $P(B, t)$ are two unique increasing continuous processes $\widetilde{P}(B, t) \in \mathscr{A}_{\text {loc }}^{+}\left(\mathscr{F}_{t}, P\right)$ and $\tilde{P}^{x}(B, t) \in \mathscr{A}_{\text {loc }}^{+}\left(\mathscr{F}_{t}^{x}, P\right)$ such that

$$
Q(B, t)=P(B, t)-\widetilde{P}(B, t) \in \mathscr{M}_{\mathrm{loc}}^{2}\left(\mathscr{F}_{t}, P\right)
$$

and $Q^{x}(B, t)=P(B, t)-\tilde{P}^{x}(B, t) \in \mathscr{M}_{\text {loc }}^{2}\left(\mathscr{F}_{t}^{x}, P\right)$. Furthermore,

$$
\left\langle Q\left(B_{1}, t\right), Q\left(B_{2}, t\right)\right\rangle=\tilde{P}\left(B_{1} \cap B_{2}, t\right),
$$

and

$$
\left\langle Q^{x}\left(B_{1}, t\right), Q^{x}\left(B_{2}, t\right)\right\rangle=\widetilde{P}^{x}\left(B_{1} \cap B_{2}, t\right) .
$$

Finally, the functions $P, \widetilde{P}, \widetilde{P}^{x}, Q, Q^{x}$ considered as random set functions on $\mathscr{Z}$ are countably additive. 
Note. The condition that the $T_{n}$ are totally inaccessible is equivalent to the assertion that the $\widetilde{P}(B, t)$ are continuous. See [4] for alternative conditions.

A real-valued function $f(z, t)=f(z, \omega, t)$ is said to be predictable, and one writes $f \in \mathscr{P}\left(\mathscr{F}_{t}\right)$, if it is measurable with respect to $\mathscr{Z} \otimes \mathscr{F} \otimes \mathscr{B}$ and if for each fixed $z, f(z, \cdot, \cdot)$ is predictable in the sense of $\S 2.4$ above. The family $\mathscr{P}\left(\mathscr{F}_{t}^{x}\right)$ is defined similarly. If $f \in \mathscr{P}\left(\mathscr{F}_{t}\right)$, respectively $\mathscr{P}\left(\mathscr{F}_{t}^{x}\right)$, we call $f$ a $\mathscr{F}_{t}$-predictable, respectively $\mathscr{F}_{t}^{x}$-predictable, process. The following classes of predictable functions are used in the martingale representation results:

$$
\begin{aligned}
L^{2}\left(\tilde{P}^{x}\right) & =\left\{f \in \mathscr{P}\left(\mathscr{F}_{t}^{x}\right) \mid\left(\|f\|_{2}\right)^{2}=E \int_{Z} \int_{R_{+}} f^{2}(z, t) \tilde{P}^{x}(d z, d t)<\infty\right\}, \\
L^{1}\left(\tilde{P}^{x}\right) & =\left\{f \in \mathscr{P}\left(\mathscr{F}_{t}^{x}\right)\left|\|f\|_{1}=E \int_{Z} \int_{R_{+}}\right| f(z, t) \mid \tilde{P}^{x}(d z, d t)<\infty\right\}, \\
L^{1}(P) & =\left\{f \in \mathscr{P}\left(\mathscr{F}_{t}^{x}\right)\left|\|f\|_{1}=E \int_{Z} \int_{R_{+}}\right| f(z, t) \mid P(d z, d t)<\infty\right\}, \\
L^{1}\left(Q^{x}\right) & =L^{1}(P) \cap L^{1}\left(\tilde{P}^{x}\right) .
\end{aligned}
$$

It turns out that $\|f\|_{1}=\|f\|_{1}^{\tilde{1}}$, hence $L^{1}\left(\tilde{P}^{x}\right)=L^{1}(P)=L^{1}\left(Q^{x}\right)$.

$$
\begin{gathered}
L_{\text {loc }}^{2}\left(\widetilde{P}^{x}\right)=\left\{f \in \mathscr{P}\left(\mathscr{F}_{t}^{x}\right) \mid \text { there exists a sequence of s.t.s } S_{k} \rightarrow \infty\right. \text { such that } \\
\left.f(z, t) I_{\left\{t \leqq S_{k}\right\}} \in L^{2}\left(\tilde{P}^{x}\right) \text { for each } k\right\} .
\end{gathered}
$$

The classes $L_{\text {loc }}^{1}(P)$ etc. are defined in a similar manner. Evidently,

$$
L_{\mathrm{loc}}^{1}\left(Q^{x}\right)=L_{\mathrm{loc}}^{1}(P)=L_{\mathrm{loc}}^{1}\left(\widetilde{P}^{x}\right)
$$

Let $f(z, \omega, t)$ be a function which is measurable with respect to $\mathscr{Z} \otimes \mathscr{F}^{x} \otimes \mathscr{B}$ such that $f(z, \cdot, t)$ is $\mathscr{F}_{t}^{x}$-measurable for fixed $z$ and such that

$$
E \int_{Z} \int_{R_{+}}|f(z, t)| \tilde{P}^{x}(d z, d t)<\infty .
$$

Then there exists a $\mathscr{F}_{t}^{x}$-predictable function $\hat{f}$ such that

$$
E \int_{Z} \int_{R_{+}}|f-\hat{f}| \tilde{P}^{x}(d z, d t)=0
$$

This result follows easily from [22, $\S \mathrm{V}$, Thm. 23]. The result will be used in $\S 4$, $\S 5$ in the following context: Let $f \in \mathscr{P}\left(\mathscr{F}_{t}\right)$ and let $\hat{f}(z, t)=E\left(f(z, t) \mid \mathscr{F}_{t}^{x}\right)$; it can then be assumed without loss of generality that $\hat{f}$ is $\mathscr{F}_{t}^{x}$-predictable.

2.10. Representation of $\mathscr{M}^{2}\left(\mathscr{F}_{t}^{x}\right)$. For each $f \in L^{2}\left(Q^{x}\right)$ there exists a unique process $\left(f \circ Q^{x}\right)_{t} \in \mathscr{M}^{2}\left(\mathscr{F}_{t}^{x}\right)$ such that for all $g \in L^{2}\left(Q^{x}\right), \alpha$ and $\beta$ in $R$,

$$
\begin{gathered}
(\alpha f+\beta g) \circ Q^{x} \equiv \alpha\left(f \circ Q^{x}\right)+\beta\left(g \circ Q^{x}\right) \\
\left\langle f \circ Q^{x}, g \circ Q^{x}\right\rangle_{t}=\int_{Z} \int_{0}^{t} f(z, s) g(z, s) \tilde{P}^{x}(d z, d s) .
\end{gathered}
$$


Conversely if $m_{t} \in \mathscr{M}^{2}\left(\mathscr{F}_{t}^{x}\right)$, then there exists $f \in L^{2}\left(Q^{x}\right)$ such that

$$
m_{t}=\left(f \circ Q^{x}\right)_{t} \text {. }
$$

Similarly, $m_{t} \in \mathscr{M}_{\mathrm{loc}}^{2}\left(\mathscr{F}_{t}^{x}\right)$ if and only if there exists $f \in L_{\mathrm{loc}}^{2}\left(Q^{x}\right)$ such that

$$
m_{t}=\left(f \circ Q^{x}\right)_{t} .
$$

2.11. Representation of $\mathscr{M}_{\text {loc }}^{1}\left(\mathscr{F}_{t}^{x}\right)$. If $f \in L^{1}\left(P^{x}\right)$, then $\left(f \circ Q^{x}\right)_{t} \in \mathscr{M}^{1}\left(\mathscr{F}_{t}^{x}\right) \cap \mathscr{A}$, where

$$
\begin{aligned}
\left(f \circ Q^{x}\right)_{t}= & \int_{Z} \int_{0}^{t} f(z, s) Q^{x}(d z, d s)=\int_{Z} \int_{0}^{t} f(z, s) P(d z, d s) \\
& -\int_{Z} \int_{0}^{t} f(z, s) \tilde{P}^{x}(d z, d s)
\end{aligned}
$$

the integrals on the right being Stieltjes integrals. Conversely if $m_{t} \in \mathscr{M}^{1}\left(\mathscr{F}_{t}^{x}\right) \cap \mathscr{A}$, then there is $f \in L^{1}\left(\tilde{P}^{x}\right)$ such that

$$
m_{t}=\left(f \circ Q^{x}\right)_{t} .
$$

Finally, $m_{t} \in \mathscr{M}_{\text {loc }}^{1}\left(\mathscr{F}_{t}^{x}\right)$ if and only if there is $f \in L_{\text {loc }}^{1}\left(\widetilde{P}^{x}\right)$ such that

$$
m_{t}=\left(f \circ Q^{x}\right)_{t}=\int_{Z} \int_{0}^{t} f(z, s)\left[P(d z, d s)-\widetilde{P}^{x}(d z, d s)\right]
$$

Remark 2.1.1. If $m_{t} \in \mathscr{M}_{\mathrm{loc}}^{1}\left(\mathscr{F}_{t}^{x}\right)$ has continuous sample paths, then $m_{t} \equiv 0$.

2. If more than one representation above applies then the representations coincide.

2.12. Local description of a fundamental process. Let $\left(x_{t}, \mathscr{F}_{t}, P\right)$ be a fundamental process with values in $(Z, \mathscr{Z})$, and consider the increasing processes $\widetilde{P}(B, t)$ and $\widetilde{P}^{x}(B, t)$. Let $\Lambda(t)=\widetilde{P}(Z, t), \Lambda^{x}(t)=\widetilde{P}^{x}(Z, t)$. The countable additivity of these functions with respect to $B \in \mathscr{Z}$ implies that there exist predictable processes $n(B, t)$ and $n^{x}(B, t)$ such that for all $B \in \mathscr{Z}$,

$$
\begin{gathered}
\tilde{P}(B, t)=\int_{0}^{t} n(B, s) \Lambda(d s), \\
\tilde{P}^{x}(B, t)=\int_{0}^{t} n^{x}(B, s) \Lambda^{x}(d s) .
\end{gathered}
$$

Evidently it can be assumed that $n(Z, s)=n^{x}(Z, s) \equiv 1$. The system $\{n(B, t), \Lambda(t)\}$ or $\{n(d z, t), \Lambda(d t)\}$ is analogous to a Lévy system for a Hunt process [5]. The system $\{n(d z, t), \Lambda(d t)\}$ will be called an extrinsic local description of $x$, whereas $\left\{n^{x}(d z, t), \Lambda^{x}(d t)\right\}$ is called the intrinsic local description of $x$, because of the following interpretation: the probability that $x$ has a jump in $[t, t+d t]$ given $\mathscr{F}_{t}$, (respectively $\mathscr{F}_{t}^{x}$ ) is $\Lambda(d t)+o(d t)$ (respectively $\Lambda^{x}(d t)+o(d t)$ ), while $n(B, t)$, (respectively $n^{x}(B, t)$ ) is the probability that $x_{t} \in B$ given $\mathscr{F}_{t}\left(\mathscr{F}_{t}^{x}\right)$ and given that a jump occurs at $t$. For future reference we note the following trivial but important fact. 
Fact. Let $\{n(B, t), \Lambda(t)\}$ and $\left\{n^{x}(B, t), \Lambda^{x}(t)\right\}$ be extrinsic and intrinsic local descriptions. Then for all $B \in \mathscr{Z}$, and $t \in R_{+}$,

$$
E\left\{\int_{0}^{t} n(B, s) \Lambda(d s) \mid \mathscr{F}_{t}^{x}\right\}=\int_{0}^{t} n^{x}(B, s) \Lambda^{x}(d s) \quad \text { a.s. }
$$

2.13. Fundamental example. The results in the succeeding sections will be specialized to the following example which covers many practical cases such as Poisson, counting, birth and death, and queueing processes.

Let $\left(x_{t}, \mathscr{F}_{t}, P\right)$ be a fundamental process with values in $(Z, \mathscr{Z})$. Suppose that from each $z \in Z$ the process can make at most $n$ transitions, where $n$ is a fixed finite number. Thus the transitions can be represented by a "state-transition" diagram of Fig. 1, where the transitions are labeled $\sigma_{1}, \cdots, \sigma_{n}$. Define the counting processes $p_{i}(t), 1 \leqq i \leqq n$,

$p_{i}(t)=$ number of transitions of type $i$ made by the process $x_{t}$ prior to $t$.

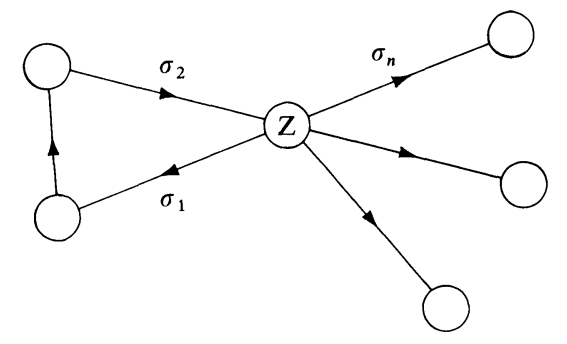

FIG. 1. State-transition diagram for fundamental example

Then there exist increasing processes $\tilde{p}_{i}(t)$ and $\tilde{p}_{i}^{x}(t)$ such that

$$
\begin{aligned}
& q_{i}(t)=p_{i}(t)-\tilde{p}_{i}(t) \in \mathscr{M}_{\mathrm{loc}}^{2}\left(\mathscr{F}_{t}, P\right), \\
& q_{i}^{x}(t)=p_{i}(t)-\tilde{p}_{i}^{x}(t) \in \mathscr{M}_{\mathrm{loc}}^{2}\left(\mathscr{F}_{t}^{x}, P\right) .
\end{aligned}
$$

Furthermore, $m_{t} \in \mathscr{M}^{2}\left(\mathscr{F}_{t}^{x}, P\right)$, respectively $\mathscr{M}_{\text {loc }}^{2}\left(\mathscr{F}_{t}^{x}, P\right)$, if and only if there exist $f_{i} \in L^{2}\left(\tilde{p}_{i}^{x}\right)$, respectively $L_{\mathrm{loc}}^{2}\left(\tilde{p}_{i}^{x}\right)$, such that

$$
m_{t}=\sum_{i=1}^{n}\left(f_{i} \circ q_{i}^{x}\right)_{t}
$$

and $m_{t} \in \mathscr{M}^{1}\left(\mathscr{F}_{t}^{x}, P\right) \cap \mathscr{A}$, respectively $\mathscr{M}_{\text {loc }}^{1}\left(\mathscr{F}_{t}^{x}, P\right)$, if and only if there exist $f_{i} \in L^{1}\left(\tilde{p}_{i}^{x}\right)$, respectively $L_{\mathrm{loc}}^{1}\left(\tilde{p}_{i}^{x}\right)$, such that

$$
m_{t}=\sum_{i=1}^{n}\left(f_{i} \circ q_{i}^{x}\right)_{t}
$$

where the integral is a Stieltjes integral.

We call $\left(\tilde{p}_{i}, \cdots, \tilde{p}_{n}\right)$, respectively $\left(\tilde{p}_{i}^{x}, \cdots, \tilde{p}_{n}^{x}\right)$, the extrinsic, respectively intrinsic, local descriptions. 
Remark 2.2. If $\left(x_{t}, \mathscr{F}_{t}, P\right)$ is a counting process, ${ }^{3}$ then Fig. 1 simplifies to Fig. 2 and there is only one transition. Hence in this case $n=1$ in (2.5) and (2.6).

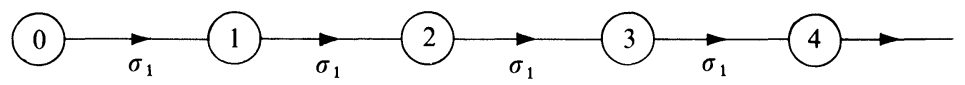

FIG. 2. State-transition diagram for counting processes

For this special case Brémaud [6] has obtained the representation for $\mathscr{M}_{\text {loc }}^{2}\left(\mathscr{F}_{t}^{x}\right)$, whereas Davis [7] has extended it to the class $\mathscr{M}_{\text {loc }}^{1}\left(\mathscr{F}_{t}^{x}\right)$. However both these results were obtained only for the case where the law of $\left(x_{t}, \mathscr{F}_{t}^{x}, P\right)$ is mutually absolutely continuous with respect to the law for a standard Poisson process (see $\S 3$ ).

3. Solutions to specified local descriptions by change of law. In $\S 3.1$ we present a very useful technique for transforming one fundamental process $\left(x_{t}, \mathscr{F}_{t}, P\right)$ with a l.d. (local description) $(n, \Lambda)$ to another process with a different prespecified 1.d. The questions of uniqueness of the solution is discussed in $\S 3.2$. Section 3.3 consists of some sufficient conditions which guarantee that the technique is applicable. Finally $\S 3.4$ presents a class of processes which can be transformed into other processes with this technique.

Let $\left(x_{t}, \mathscr{F}_{t}^{x}, P\right)$ be a fundamental process with values in $(Z, \mathscr{Z})$ and with intrinsic local description (1.d.) $\left(n^{x}(d z, t), \Lambda^{x}(d t)\right)$ so that

$$
\tilde{P}^{x}(B, t)=\int_{B} \int_{0}^{t} n^{x}(d z, s) \Lambda^{x}(d s), \quad t \in R_{+} .
$$

Since we will be only dealing with the "intrinsic" $\sigma$-field $\mathscr{F}_{t}^{x}$ in this section, the superscript $x$ will be omitted here. Hence $\mathscr{F}_{t}=\mathscr{F}_{t}^{x}, \widetilde{P}=\widetilde{P}^{x}$ etc.

3.1. The transformation technique. Let $P_{1}$ be another probability measure on $(\Omega, \mathscr{F})$ and suppose that

$$
P_{1} \ll P,
$$

i.e., $P_{1}$ is absolutely continuous with respect to $P$. It is evident that the same function $x_{t}(\omega)$ defines another fundamental process $\left(x_{t}, \mathscr{F}_{t}, P_{1}\right)$ with a possibly different 1.d. $\left(n_{1}(B, t), \Lambda_{1}(t)\right)$ say. We are going to determine the relationship between the two descriptions.

Let $L=d P_{1} / d P$ be the Radon-Nikodym derivative. The r.v. $L \geqq 0$ and $E(L)^{4}=1$. Let $L_{t}=E\left(L \mid \mathscr{F}_{t}\right)$. Then $\left(L_{t}, \mathscr{F}_{t}, P\right)$ is a uniformly integrable martingale, $\lim _{t \rightarrow \infty} L_{t}=L$ a.s. and in $L^{1}$ by [3, remark after $\S$ VI, Thm. 6].

Proposition 3.1. (i) If $L>0$ a.s. $P$, then for almost all $\omega, L_{t^{-}}(\omega)>0$ and $L_{t}(\omega)>0$ for all $t$.

(ii) Let

$$
T(\omega)=\inf \left\{t \mid L_{t^{-}}(\omega)=0 \text { or } L_{t}(\omega)=0\right\}
$$

\footnotetext{
${ }^{3} \mathrm{~A}$ counting process is an integer-valued process which starts at 0 and has unit jumps.

${ }^{4} E, E_{1}$, denotes expectation with respect to $P, P_{1}$.
} 
Then for almost all $\omega, L_{t}(\omega)=0$ for $t \geqq T(\omega)$.

Proof. (i) Clearly $L>0$ a.s. implies $L_{t}>0$ a.s. and then the second part of the assertion follows from (ii), and the latter follows from [3, § VI, Thm. 15].

Remarks 3.1. (i) If $L>0$ a.s. $P$, then in fact $P \ll P_{1}$, i.e., the two measures are mutually absolutely continuous.

(ii) It is easy to give examples such that $L_{t}>0$ for all $t$ but $P(L=0)>0$.

For $\varepsilon>0$ let

$$
T_{\varepsilon}(\omega)=\inf \left\{t \mid L_{t^{-}}(\omega) \leqq \varepsilon\right\}
$$

Proposition 3.2. $T_{\varepsilon}$ is a s.t. for all $\varepsilon$ and

$$
\lim _{\varepsilon \rightarrow 0} T_{\varepsilon}(\omega)=T(\omega) \text { a.s. } P
$$

Proof. The fact that $T_{\varepsilon}$ is a s.t. follows from the fact that the process $L_{t^{-}}$is left-continuous and from $\left[3, \S \mathrm{IV}\right.$, Thm. 52]. Now $T_{\varepsilon}$ is clearly nondecreasing with $\varepsilon$. Let

$$
T_{0}(\omega)=\lim _{\varepsilon \rightarrow 0} T_{\varepsilon}(\omega)
$$

Suppose $T(\omega)=\infty$ and per contra $T_{0}(\omega)<\infty$. Then there exists a sequence $t_{i}$ increasing to $t_{0}<\infty$ such that $L_{t_{i}}-(\omega) \rightarrow 0$. By left-continuity $L_{t_{0}-}(\omega)=0$ and so $T(\omega) \leqq t_{0}$. Next suppose $T(\omega)<\infty$. By Proposition 3.1, for almost all such $\omega$, $T_{0}(\omega) \leqq T(\omega)$. If $T_{0}(\omega)<T(\omega)$, then a repetition of the previous argument will end in a contradiction. Once again $T_{0}(\omega)=T(\omega)$.

For $\varepsilon>0$ let

$$
L_{t}^{\varepsilon}(\omega)=L_{t \wedge T \varepsilon}(\omega), \quad t \in R_{+}
$$

Then $L_{t}^{\varepsilon}-L_{0} \in \mathscr{M}^{1}(P)$ and $L_{t^{-}}^{\varepsilon} \geqq \varepsilon$ for all $t$. By $\S 2.11$ there is a predictable function $f^{\varepsilon}(z, t) \in L_{\text {loc }}^{1}(P)$ such that

$(3.3)^{5} \quad L_{t}^{\varepsilon}=1+\int_{Z} \int_{0}^{t} f^{\varepsilon}(z, s) Q(d z, d s)=\int_{Z} \int_{0}^{t} f^{\varepsilon}(z, s)[P(d z, d s)-\widetilde{P}(d z, d s)]$.

Since $1 / L_{t^{-}}^{\varepsilon} \leqq 1 / \varepsilon$, therefore the process

$$
\phi^{\varepsilon}(z, s)=\left[f^{\varepsilon}(z, s)\right] / L_{s^{-}}^{\varepsilon} \in L_{\text {loc }}^{1}(\widetilde{P})
$$

and hence

$$
m^{\varepsilon}(t)=\int_{Z} \int_{0}^{t} \phi^{\varepsilon}(z, s) Q(d z, d s) \in \mathscr{M}_{\mathrm{loc}}^{1}\left(\mathscr{F}_{t}, P\right)
$$

which upon substitution into (3.3) gives

$$
L_{t}^{\varepsilon}=1+\int_{0}^{t} L_{s^{-}}^{\varepsilon} d m_{s}^{\varepsilon}
$$

${ }^{5}$ Here it is being assumed that $L_{0} \equiv 1$ which is indeed the case if $\mathscr{F}_{0}$ is trivial. Otherwise, in the sequel, replace the martingale $L_{t}$ by $L_{t} / L_{0}$. 
By the exponentiation formula of $\S 2.8$,

$$
L_{t}^{\varepsilon}=\mathscr{E}\left(m_{t}^{\varepsilon}\right)=\exp \left(m_{t}^{\varepsilon}-\frac{1}{2}\left\langle m^{\varepsilon, c}, m^{\varepsilon, c}\right\rangle_{t}\right) \prod_{s \leqq t}\left(1+\Delta m_{s}^{\varepsilon}\right) e^{-\Delta m_{s}^{\varepsilon}}
$$

By the Remark in $\S 2.11, m^{\varepsilon, c} \equiv 0$, hence (3.5) simplifies to

$$
L_{t}^{\varepsilon}=\exp \left(m_{t}^{\varepsilon}\right) \prod_{s \leqq t}\left(1+\Delta m_{s}^{\varepsilon}\right) e^{-\Delta m_{s}^{\varepsilon}}
$$

Rewriting (3.4) as

$$
m^{\varepsilon}(t)=\int_{Z} \int_{0}^{t} \phi^{\varepsilon}(z, s) P(d z, d s)-\int_{Z} \int_{0}^{t} \phi^{\varepsilon}(z, s) \widetilde{P}(d z, d s)
$$

and acknowledging that the second integral has continuous sample paths (since $\widetilde{P}$ is continuous) it follows that for almost all $\omega$,

$$
\Delta m_{s}^{\varepsilon}(\omega)=m_{s}^{\varepsilon}(\omega)-m_{s^{-}}^{\varepsilon}(\omega)=\int_{Z} \phi^{\varepsilon}(z, s)(\omega)\left[P(d z, s)(\omega)-P\left(d z, s^{-}\right)(\omega)\right] .
$$

Also since $P(B, s)(\omega)-P\left(B, s^{-}\right)(\omega)$ equals 1 or 0 depending upon whether or not $x_{s^{-}}(\omega) \neq x_{s}(\omega)$ and $x_{s}(\omega) \in B$, therefore the term $\left(1+\Delta m_{s}^{\varepsilon}\right)$ in (3.6) can be written as

$$
\left(1+\Delta m_{s}^{\varepsilon}\right)(\omega)=\int_{z}\left(1+\phi^{\varepsilon}(z, s)(\omega)\right)\left[P(d z, s)(\omega)-P\left(d z, s^{-}\right)(\omega)\right] .
$$

From (3.8), (3.9) it follows respectively that

$$
\begin{gathered}
\sum_{s \leqq t} \Delta m_{s}^{\varepsilon}(\omega)=\sum_{\substack{s \leqq t \\
x_{s}-\neq x_{s}}} \phi^{\varepsilon}\left(x_{s}(\omega), s\right), \\
\prod_{s \leqq t}\left(1+\Delta m_{s}^{\varepsilon}(\omega)\right)=\prod_{\substack{s \leq t \\
x_{s}-\neq x_{s}}}\left(1+\phi^{\varepsilon}\left(x_{s}(\omega), s\right)\right),
\end{gathered}
$$

which upon substitution, together with (3.7), into (3.6), yields after some cancellation the first interesting result:

$$
L_{t}^{\varepsilon}=\prod_{\substack{s \leq t \\ x_{s}-\neq x_{s}}}\left[1+\phi^{\varepsilon}\left(x_{s}, s\right)\right] \exp \left(-\int_{z} \int_{0}^{t} \phi^{\varepsilon}(z, s) \tilde{P}(d z, d s)\right) .
$$

Finally let $\varepsilon_{k}>0, k=1,2, \cdots$, be a sequence decreasing to 0 , let $S_{0}=0$, $S_{k}=T_{\varepsilon_{k}}, k=1,2, \cdots$, and let

$$
\left.\phi(z, s)=\sum_{k=1}^{\infty} \phi^{\varepsilon_{k}}(z, s) I_{\left\{S_{k-1}<s \leqq\right.} S_{k}\right\} .
$$

$\phi$ is predictable since $\phi^{\varepsilon_{k}}$ is predictable and $I_{\left\{S_{k-1}<s \leqq S_{k}\right\}}$ is left-continuous. Since by definition, $L_{t}^{\varepsilon}=L_{t \wedge T_{\varepsilon}}^{\varepsilon^{\prime}}$ for $\varepsilon^{\prime}<\varepsilon$, we have proved the following result.

THEOREM 3.1. Let $P_{1} \ll P$, and let $L_{t}=E\left(d P_{1} / d P \mid \mathscr{F}_{t}^{x}\right)$. Let

$$
T=\inf \left\{t \mid L_{t^{-}}=0 \text { or } L_{t}=0\right\} .
$$


Then there exists a predictable function $\phi(z, s)$ and an increasing sequence $S_{k}$ of s.t.s converging to $T$ such that

$$
\phi_{k}(z, s)=\phi(z, s) I_{\left\{s \leqq S_{k}\right\}} \in L_{1 \mathrm{loc}}^{1}(\widetilde{P})
$$

and

$$
L_{t \wedge S_{k}}=\prod_{\substack{s \leqq t \\ x_{s^{-}} \neq x_{s}}}\left[1+\phi_{k}\left(x_{s}, s\right)\right] \exp \left[-\int_{Z} \int_{0}^{t} \phi_{k}(z, s) \tilde{P}(d z, d s)\right] .
$$

The product on the right converges a.s. whereas the integral is a Stieltjes integral.

Remarks 3.2. (i) If $L=d P_{1} / d P>0$ a.s., then $T=\infty$ a.s. so that the result above implies that $\phi \in L_{\text {loc }}^{1}(\widetilde{P})$. However if this is not the case then it is not true that in general $\phi \in L_{\mathrm{loc}}^{1}$. Some additional properties of $\phi$ are given in Theorem 3.2 below. Nevertheless, very loosely speaking, one can interpret (3.11) as

$$
L_{t}=\prod_{\substack{s \leqq t \\ x_{s}-\neq x_{s}}}\left[1+\phi\left(x_{s}, s\right)\right] \exp \left[-\int_{Z} \int_{0}^{t} \phi(z, s) \tilde{P}(d z, d s)\right] \text { for } t<T
$$

Indeed some such loose interpretation has to be used in understanding the corresponding formulas of [6], [23].

(ii) The characterization (3.11) has been derived earlier [23], [24] for the case where $\left(x_{t}, \mathscr{F}_{t}, P\right)$ is a Brownian motion. The techniques for the proof are identical except that in deriving (3.4) one observes that every martingale on a Brownian motion sample space is a stochastic integral of the Brownian motion (see [5]), and that all martingales are continuous so that (3.5) simplifies to

$$
L_{t}^{\varepsilon}=\exp \left(m_{t}^{\varepsilon}-\frac{1}{2}\left\langle m^{\varepsilon, c}, m^{\varepsilon, c}\right\rangle_{t}\right) .
$$

(iii) For the fundamental example the representation (3.11) becomes, using $\S 2.12$,

$$
L_{t \wedge S_{k}}=\prod_{i=1}^{n}\left\{\prod_{\substack{s \leqq t \\\left(x_{s}-, x_{s}\right) \in \sigma_{i}}}\left[1+\phi_{k}^{i}(s)\right] \exp \left[-\int_{0}^{t} \phi_{k}^{i}(s) \tilde{p}_{i}^{x}(d s)\right]\right\}
$$

for some predictable $\phi^{i}(s), 1 \leqq i \leqq n$, such that $\phi_{k}^{i} \in L_{\mathrm{loc}}^{1}\left(\tilde{p}_{i}^{x}\right)$. Here the notation $\left(x_{s^{-}}, x_{s}\right) \in \sigma_{i}$ means that $x$ makes a transition of type $i$ at time $s$.

If $\left(x_{t}, \mathscr{F}_{t}^{x}, P\right)$ is a Poisson process then in the above $n=1$ and, as is well known, $\tilde{p}_{i}^{x}(d s) \equiv d s$. For this case the result was first obtained by Brémaud [6] without the integrability condition on $\phi$, and for the case $L>0$ a.s., by Van Schuppen [24], and by Davis [7] who proves in addition that then $\phi \in L_{\text {loc }}^{1}$. Brémaud [6] also obtains this representation for the case where the example is a Markov chain.

We proceed to obtain the relations between the local descriptions. The next result is well known.

LeMma 3.1. $m_{t} \in \mathscr{M}_{\mathrm{loc}}^{1}\left(\mathscr{F}_{t}, P_{1}\right)$ if and only if $m_{t} L_{t} \in \mathscr{M}_{\mathrm{loc}}^{1}\left(\mathscr{F}_{t}, P\right)$.

Proof. Let $S_{k} \rightarrow \infty$ be a sequence of s.t.s such that for each $k$,

$$
m_{t \wedge S_{k}} L_{t \wedge S_{k}} I_{\left\{S_{k}>0\right\}} \in \mathscr{M}^{1}(P)
$$


First of all,

$$
\begin{aligned}
& E_{1}\left|m_{t \wedge S_{k}} I_{\left\{S_{k}>0\right\}}\right|=E L\left|m_{t \wedge S_{k}} I_{\left\{S_{k}>0\right\}}\right| \\
& =E L_{t \wedge S_{k}}\left|m_{t \wedge S_{k}} I_{\left\{S_{k}>0\right\}}\right| \quad(\text { by (3.13)) } \\
& <\infty \text {. }
\end{aligned}
$$

Next for $s \leqq t$,

$$
E_{1}\left(m_{t \wedge S_{k}} I_{\left\{S_{k}>0\right\}} \mid \mathscr{F}_{s}\right)=\frac{E\left(m_{t \wedge S_{k}} L_{t \wedge S_{k}} I_{\left\{S_{k}>0\right\}} \mid \mathscr{F}_{s}\right)}{E\left(L_{t \wedge S_{k}} \mid \mathscr{F}_{s}\right)} .
$$

From (3.13) and the fact that $L_{t} \in \mathscr{M}^{1}(P)$ the right-hand side simplifies to

$$
\frac{m_{s \wedge S_{k}} L_{s \wedge S_{k}} I_{\left\{S_{k}>0\right\}}}{L_{s \wedge S_{k}}}=m_{s \wedge S_{k}} I_{\left\{S_{k}>0\right\}},
$$

which proves the "if" part of the assertion.

Conversely suppose that

$$
m_{t \wedge S_{k}} I_{\left\{S_{k}>0\right\}} \in \mathscr{M}^{1}\left(P_{1}\right) .
$$

It will be shown that for $s \leqq t$,

$$
E\left(L_{t \wedge S_{k}} m_{t \wedge S_{k}} I_{\left\{S_{k}>0\right\}} \mid \mathscr{F}_{s}\right)=L_{s \wedge S_{k}} I_{\left\{S_{k}>0\right\}} \quad \text { a.s. } P .
$$

So let $A \in \mathscr{F}_{s}$. Then

which proves (3.15).

$$
\begin{aligned}
E\left(I_{A} L_{t \wedge S_{k}} m_{t \wedge S_{k}} I_{\left\{S_{k}>0\right\}}\right) & =E_{1}\left(I_{A} m_{t \wedge S_{k}} I_{\left\{S_{k}>0\right\}}\right) \\
& =E_{1}\left(I_{A} m_{s \wedge S_{k}} I_{\left\{S_{k}>0\right\}}\right) \quad(\text { by }(3.14)) \\
& =E\left(L_{s \wedge S_{k}} I_{A} m_{s \wedge S_{k}} I_{\left\{S_{k}>0\right\}}\right),
\end{aligned}
$$

THEOREM 3.2. Let $\left(x_{t}, \mathscr{F}_{t}, P\right)$ be a fundamental process with values in $(Z, \mathscr{Z})$ and with (intrinsic) l.d. $(n(d z, t), \Lambda(d t))$. Let $P_{1} \ll P$ and let $L_{t}=E\left(d P_{1} / d P \mid \mathscr{F}_{t}\right)$ have the representation (3.11). Then $\left(x_{t}, \mathscr{F}_{t}, P_{1}\right)$ has l.d. $\left(n_{1}(d z, t), \Lambda_{1}(d t)\right)$, where

$$
\Lambda_{1}(t)=\Lambda(d t) \quad \text { and } \quad n_{1}(d z, t)=(1+\phi(z, t)) n(d z, t) .
$$

Furthermore, it can be assumed that

$$
(1+\phi) \geqq 0 \text { and }(1+\phi) \in L_{\mathrm{loc}}^{1}(\tilde{P})
$$

with respect to probability measure $P_{1}$.

Proof. By $\S 2.9$ there exist continuous increasing processes $\widetilde{P}_{1}(B, t) \in \mathscr{A}_{\mathrm{loc}}^{+}\left(P_{1}\right)$ such that

$$
Q_{1}(B, t)=P(B, t)-\widetilde{P}_{1}(B, t) \in \mathscr{M}_{\mathrm{loc}}^{2}\left(P_{1}\right) .
$$

Hence to show (3.16) it is equivalent to prove that

$$
\tilde{P}_{1}(B, t)=\int_{B} \int_{0}^{t}(1+\phi(z, s)) \tilde{P}(d z, d s) .
$$


Let $S_{i}, \phi_{i}$ be as in Theorem 3.1, and let

$$
\begin{gathered}
Q_{1}^{i}(B, t)=Q_{1}\left(B, t \wedge S_{i}\right)=P\left(B, t \wedge S_{i}\right)-\widetilde{P}_{1}\left(B, t \wedge S_{i}\right), \\
m_{t}=P\left(B, t \wedge S_{i}\right)-\int_{B} \int_{0}^{t}\left(1+\phi_{i}(z, s)\right) \widetilde{P}(d z, d s) .
\end{gathered}
$$

It will be shown first that $m_{t} \in \mathscr{M}_{\text {loc }}^{1}\left(P_{1}\right)$. By Lemma 3.1 it is enough to show that

$$
L_{t} m_{t} \in \mathscr{M}_{\mathrm{loc}}^{1}(P) \text {. }
$$

Since $\phi_{i} \in L_{\text {loc }}^{1}(P)$, therefore $m_{t}$ is in $\mathscr{S}_{\text {loc }}(P)$, also $L_{t} \in \mathscr{M}^{1}(P) \subset \mathscr{S}_{\text {loc }}(P)$. Hence one can apply the differential formula of $\S 2.7$ to obtain

$$
L_{t} m_{t}=\int_{0}^{t} m_{s^{-}} d L_{s}+\int_{0}^{t} L_{s^{-}} d m_{s}+\sum_{s \leqq t}\left[\Delta\left(m_{s} L_{s}\right)-m_{s^{-}} \Delta L_{s}-L_{s^{-}} \Delta m_{s}\right]
$$

From (3.21),

$$
\int_{0}^{t} L_{s^{-}} d m_{s}=\int_{0}^{t \wedge s_{i}} L_{s^{-}} P(B, d s)-\int_{B} \int_{0}^{t \wedge s_{i}} L_{s^{-}}(1+\phi) \widetilde{P}(d z, d s),
$$

and since $\Delta\left(m_{s} L_{s}\right)=\left(m_{s^{-}}+\Delta m_{s}\right)\left(L_{s^{-}}+\Delta L_{s}\right)-m_{s^{-}} L_{s^{-}}=m_{s^{-}} \Delta L_{s}+L_{s^{-}} \Delta m_{s}$ $+\Delta L_{s} \Delta m_{s}$, therefore the last term in (3.22) equals

$$
\sum_{s \leqq t} \Delta L_{s} \Delta m_{s}=\int_{B} \int_{0}^{t \wedge s_{i}} L_{s^{-}} \phi(z, s) P(d z, d s)
$$

from (3.11) and (3.21). Substituting these relations back into (3.22) gives

$$
\begin{aligned}
L_{t} m_{t} & =\int_{0}^{t} m_{s^{-}} d L_{s}+\int_{B} \int_{0}^{t \wedge s_{i}} L_{s^{-}}(1+\phi) P(d z, d s)-\int_{B} \int_{0}^{t \wedge s_{i}} L_{s^{-}}(1+\phi) \tilde{P}(d z, d s) \\
& =\int_{0}^{t} m_{s^{-}} d L_{s}+\int_{B} \int_{0}^{t \wedge s_{i}} L_{s^{-}}(1+\phi) Q(d z, d s),
\end{aligned}
$$

which is clearly in $\mathscr{M}_{\mathrm{loc}}^{1}(P)$. Hence $m_{t} \in \mathscr{M}_{\mathrm{loc}}^{1}\left(P_{1}\right)$. Since $Q_{1}^{i}(B, t) \in \mathscr{M}_{\mathrm{loc}}^{1}(P)$, subtracting (3.21) from (3.20) implies that

$$
\widetilde{P}_{1}\left(B, t \wedge S_{i}\right)-\int_{B} \int_{0}^{t \wedge S_{i}}(1+\phi(z, s)) \tilde{P}(d z, d s) \in \mathscr{M}_{\mathrm{loc}}^{1}\left(P_{1}\right) .
$$

But this process has continuous sample paths, hence it must vanish, i.e., for almost all $\omega\left(P_{1}\right.$ measure $)$

$$
\tilde{P}_{1}\left(B, t \wedge S_{i}\right)=\int_{B} \int_{0}^{t \wedge S_{i}}(1+\phi(z, s)) \widetilde{P}(d z, d s) \text { for all } t
$$

which proves (3.19) and thereby (3.16). The assertion contained in (3.17) follows from the fact that $\widetilde{P}_{1}$ has increasing sample paths and is in $\mathscr{A}_{\text {loc }}^{+}\left(P_{1}\right)$.

Remark 3.3. (i) It has been shown that $\phi \in L_{\text {loc }}^{1}(\widetilde{P})$ in the probability space $\left(\Omega, \mathscr{F}, P_{1}\right)$ and $n o t$ in $(\Omega, \mathscr{F}, P)$. 
(ii) The transformation of 1.d. for the case where $\left(x_{t}, P\right)$ and $\left(x_{t}, P_{1}\right)$ are both Hunt processes has been obtained in [5]. For this case the local description is called a Lévy system.

(iii) For the case of the fundamental example with 1.d. $\left(\tilde{p}_{1}, \cdots, \tilde{p}_{n}\right)$ under $P$, the l.d. under $P_{1}$ is $\left(\left(1+\phi^{1}\right) \tilde{p}_{1}, \cdots,\left(1+\phi^{n}\right) \tilde{p}_{n}\right)$, where the $\phi^{i}$ are as in Remark 3.2 (iii).

Theorems 3.1, 3.2 allow us to obtain in certain cases processes which have certain specified 1.d. from known processes with other descriptions. Put differently, we have a "synthesis" procedure for obtaining "global" solutions for a class of 1.d.s. This is summarized in the following theorem, whose proof is now immediate.

THEOREM 3.3. (Existence of solutions to local descriptions). Let $\left(x_{t}, \mathscr{F}_{t}, P\right)$ be a fundamental process with values in $(Z, \mathscr{Z})$ and with intrinsic l.d. $(n(d z, t), \Lambda(d t))$. Let $\phi(z, s)$ be a predictable function such that

$$
\phi(z, s) \in L_{\text {loc }}^{1}(\widetilde{P})
$$

and

$$
\int_{\Omega} L_{\infty} d P=1
$$

where

$$
L_{t}=\prod_{\substack{s \leqq t \\ x_{s}=\neq x_{s}}}\left[1+\phi\left(x_{s}, s\right)\right] \exp \left[-\int_{Z} \int_{0}^{t} \phi(z, s) \tilde{P}(d z, d s)\right] .
$$

Then $\left(x_{t}, \mathscr{F}_{t}, P_{1}\right)$ is a fundamental process with l.d. $\left(n_{1}(d z, t), \Lambda(d t)\right)$, where

$$
n_{1}(d z, t)=(1+\phi(z, t)) n(d z, t)
$$

and where the probability measure $P_{1}$ is given by

$$
d P_{1}=L_{\infty} d P .
$$

Remark 3.4. (i) This result is extremely useful in practice since given an arbitrary l.d. there is no way to determine whether or not there exists a process with such a description. On the other hand, from the viewpoint of dynamical processes, a 1.d. is much more natural and useful.

(ii) For the case of Brownian motion the result corresponding to the above was first obtained by Girsanov [9], and the technique was soon adopted in stochastic control problems [10], [11], [12], [13].

(iii) Bremaud [6] was the first to use this result, for the special case where $\left(x_{t}, \mathscr{F}_{t}, P\right)$ is a Poisson process, to obtain existence of several "self-exciting" counting processes $\left(x_{t}, \mathscr{F}_{t}, P_{1}\right)$. Snyder [14] and Rubin [15] introduce several jump processes through their 1.d. However they do not discuss whether or not there indeed exist processes with these descriptions. The result above can be used to solve this problem.

(iv) The condition (3.24) is a nontrivial restriction. For the Brownian motion case some sufficient conditions on the local description have been derived which guarantee (3.24). See [10], [11]. For our case similar conditions are given below in $\S 3.3$. 
(v) Theorem 3.3 does not address itself to the question of uniqueness of the solution. This question is discussed next.

3.2. Uniqueness of solutions with specified l.d. To discuss uniqueness of laws of solutions it is convenient to assume that $\Omega$ is the space of sample functions and that the process $x_{t}$ on $\Omega$ is merely the "evaluation" process, i.e., $x_{t}(\omega)=\omega_{t}$. The probability on $\Omega$ is then the law of the process. We will be dealing with two such processes, $x_{t}$ and $y_{t}$, with the same set of sample functions but with different laws. Hence we must have two different probability spaces $\left(\Omega^{x}, \mathscr{F}_{t}^{x}, P^{x}\right)$ and $\left(\Omega^{y}, \mathscr{F}_{t}^{y}, P^{y}\right)$, where $\left(\Omega^{x}, \mathscr{F}_{t}^{x}\right)$ and $\left(\Omega^{y}, \mathscr{F}_{t}^{y}\right)$ are copies of the same family $\left(\Omega, \mathscr{F}_{t}\right)$. In particular, then, $x$ and $y$ are identical functions on $\Omega \times[0,1]$.

Since we are unable to obtain any interesting results for the infinite time interval, therefore in Theorem 3.4 and Corollary 3.1, $t \in[0,1]$.

DEFINITION 3.1. An (intrinsic) l.d. $(n, \Lambda)$ is said to have unique solutions if all fundamental processes $\left(x_{t}, \mathscr{F}_{t}, P\right)$ with l.d. $(n, \Lambda)$ have the same law.

THEOREM 3.4. Let $x_{t}, y_{t}, 0 \leqq t \leqq 1$, be fundamental processes with values in $(Z, \mathscr{Z})$, and on the (sample function) spaces $\left(\Omega^{x}, \mathscr{F}_{t}^{x}, P^{x}\right),\left(\Omega^{y}, \mathscr{F}_{t}^{y}, P^{y}\right)$ respectively. Let $(n, \Lambda)$ be the l.d. of $x$ and $((1+\phi) n, \Lambda)$ the l.d. of $y$ for some predictable function $\phi$.

Suppose that $(n, \Lambda)$ has unique solutions, and suppose that for each $\varepsilon>0$ there exist $Z_{\varepsilon} \in \mathscr{Z}$ and $k_{\varepsilon}<\infty$ such that where

(i) $P^{x}\left(B_{\varepsilon}\right) \geqq 1-\varepsilon$,

(ii) $\quad \int_{Z} \int_{0}^{1}\left|\frac{\phi(z, \omega, s)}{1+\phi(z, \omega, s)}\right|\left(P^{y}(d z, d s)+\tilde{P}^{y}(d z, d s)\right) \leqq k_{\varepsilon} \quad$ for $\omega \in B_{\varepsilon}$, where these are Stieltjes integrals.

Then

$$
\int_{\Omega} l_{1}(\omega) P^{y}(d \omega)=1 \quad \text { and } \quad d P^{x}=l_{1} d P^{y}
$$

where

$$
l_{t}=\mathscr{E}\left(\left(\psi \circ Q^{y}\right)_{t}\right) \quad \text { and } \quad \psi=-\phi /(1+\phi) .
$$

Proof. The process $\left(m_{t}, \mathscr{F}_{t}^{y}, P^{y}\right)$,

$$
m_{t}=\int_{Z} \int_{0}^{t} \psi(z, s) Q^{y}(d z, d s)=\int_{Z} \int_{0}^{t} \psi(z, s)\left[P^{y}(d z, d s)-\tilde{P}^{y}(d z, d s)\right]
$$

is, by (i) and (ii), well-defined as a Stieltjes integral. Hence $m_{t} \in \mathscr{A}_{0}\left(\mathscr{F}_{t}^{y}, P^{y}\right)$ so that it is in $\mathscr{S}_{\text {loc }}\left(P^{y}\right)$. Therefore by $\S 2.9$ there is a unique process $\left(l_{t}, \mathscr{F}_{t}^{y}, P^{y}\right)$ where

$$
l_{t}=\mathscr{E}\left(m_{t}\right)
$$
functions

Let $\varepsilon_{n}>0$ be a decreasing sequence converging to 0 . Define the predictable

$$
\psi^{n}(z, \omega, s)= \begin{cases}\psi(z, \omega, s) & \text { if } z \in Z_{\varepsilon_{n}} \\ 0 & \text { otherwise }\end{cases}
$$


Because of (ii), the process $\left(m_{t}^{n}, \mathscr{F}_{t}^{y}, P^{y}\right)$, where

$$
m_{t}^{n}=\int_{Z} \int_{0}^{t} \psi^{n}(z, s) Q^{y}(d z, d s)
$$

is a bounded martingale. Hence by $\S 2.9$ the process $\left(l_{t}^{n}, \mathscr{F}_{t}^{y}, P^{y}\right)$ is a martingale, where

$$
l_{t}^{n}=\mathscr{E}\left(m_{t}^{n}\right)
$$

Furthermore from the definition of $\psi^{n}$,

$$
l_{t}^{n}(\omega)=l_{t}(\omega) \quad \text { for all } t \text { and } \omega \in B_{\varepsilon_{n}} .
$$

By Theorem 3.3 the fundamental process $\left(y_{t}, \mathscr{F}_{t}^{y}, P^{n}\right)$, where

$$
\frac{d P^{n}}{d P^{y}}=l_{1}^{n},
$$

has a 1.d. $\left(\left(1+\psi^{n}\right)(1+\phi) n, \Lambda\right)$, and by the definition of $\psi^{n}$ and $\psi$,

$$
\left(1+\psi^{n}\right)(1+\phi)(z, \omega, t)=1 \text { for all } t, \omega \in B_{\varepsilon_{n}} \text { and } z \in Z_{\varepsilon_{n}} .
$$

Since $(n, \Lambda)$ has unique solutions, it follows that

$$
\begin{aligned}
\int_{B_{\varepsilon n}} P^{n}(d \omega) & =\int_{B_{\varepsilon n}} P^{x}(d \omega) \\
& \geqq 1-\varepsilon \quad(\text { by (i)). }
\end{aligned}
$$

From (3.26), (3.27) this implies

$$
\int_{B_{\varepsilon n}} l_{1}(\omega) P^{y}(d \omega) \geqq 1-\varepsilon,
$$

and since $\varepsilon>0$ is arbitrary, the assertion follows.

Note. We must have $P^{x}(B, t)(\omega)=P^{y}(B, t)(\omega)$ and $\widetilde{P}^{y}(d z, d t)(\omega)=[1+\phi(z, t)]$ $\times n(d z, t) \Lambda(d t)$.

COROLlARY 3.1 (uniqueness). Let $\left(x_{t}, \mathscr{F}_{t}, P\right)$ be a fundamental process with values in $(Z, \mathscr{Z})$ and with l.d. $(n, \Lambda)$ which has unique solutions. Let $\phi$ be a predictable function such that

$$
\phi(z, s) \in L_{\mathrm{loc}}^{1}\left(\tilde{P}^{x}\right), \quad E\left[\mathscr{E}\left(\left(\phi \circ Q^{x}\right)_{1}\right)\right]=1 .
$$

Suppose that $\phi$ satisfies (i) and (ii) of Theorem 3.4. Then the l.d. $((1+\phi) n, \Lambda))$ has unique solutions.

Proof. By Theorem 3.3 and the hypothesis there is a solution $\left(y_{t}, \mathscr{F}_{t}^{y}, P_{1}\right)$ with 1.d. $((1+\phi) n, \Lambda)$, where $d P_{1}=\mathscr{E}\left(\left(\phi \circ Q^{x}\right)_{1}\right) d P$. 3.4,

Suppose $\left(y_{t}, \underset{F_{t}}{y}, P_{2}\right)$ is another solution with l.d. $((1+\phi) n, \Lambda)$. By Theorem

$$
d P=l_{1} d P_{1}=l_{2} d P_{2}
$$

and since $d P_{i}=L_{1} d P$ it follows that $l_{i}>0$ a.s. $P_{i}, i=1,2$. Evidently then $P_{2}=P_{1}$. 
Remark 3.5. (i) Theorem 3.4 is inspired by [9, Lemma 7] and the development there suggests how the result can be generalized.

(ii) Conditions (i) and (ii) of Theorem 3.4 are usually easy to verify in practice. Consider a special case of the fundamental example where $\left(x_{t}, \mathscr{F}_{t}^{x}, P^{x}\right)$ is a Poisson process with rate 1 . Then $Z$ is the space of integers and $y_{t}$ is then a counting process with local "intensity" rate $1+\phi(\omega, t)$. Suppose $\phi(\omega, t)$ is expressed explicitly as a function of the past of $x$, i.e., $\phi(\omega, t)=f\left(x_{[0, t]}(\omega), t\right)$. Then the conditions (i) and (ii) are satisfied if, for instance, there is an increasing function $f_{0}$ such that

$$
\left|f\left(x_{[0, t]}, t\right)\right|+\frac{1}{\left|1+f\left(x_{[0, t]}, t\right)\right|} \leqq f_{0}(N) \quad \text { when }\left|x_{t}\right| \leqq N .
$$

For a similar condition in the Brownian motion case see [11].

(iii) Corollary 3.1 extends in an obvious way to the time interval $R_{+}$. However Theorem 3.4 does not.

3.3. Sufficient conditions. Let $\left(x_{t}, \mathscr{F}_{t}, P\right)$ be a fundamental process with values in $(Z, \mathscr{Z})$ and with intrinsic description $(n(d z, t), \Lambda(d t))$. Let $\phi(z, t) \in L_{\mathrm{loc}}^{1}(\tilde{P})$ and define the process $L_{t}, t \in[0,1]$, by

$$
L_{t}=\prod_{\substack{s \leqq t \\ x_{s}-\neq x_{s}}}\left[1+\phi\left(x_{s}, s\right)\right] \exp \left[-\int_{z} \int_{0}^{t} \phi(z, s) \widetilde{P}(d z, d s)\right] .
$$

Then $L_{t}$ also satisfies

$$
d L_{t}=L_{t^{-}} d m_{t}
$$

where

$$
m_{t}=(\phi \circ Q)_{t}
$$

We assume that $1+\phi(z, t) \geqq 0$. Then $L_{t} \geqq 0, L_{t}$ is a supermartingale and

$$
E\left(L_{1}\right) \leqq 1 \text {. }
$$

The three results below state conditions on $\phi$ which guarantee

$$
E\left(L_{1}\right)=1 \text {. }
$$

The following assumption is made throughout this subsection.

Assumption 3.1. There exists an increasing function $\mu: R_{+} \rightarrow R_{+}$such that

$$
\widetilde{P}(Z, t) \leqq \mu(t) \quad \text { a.s. }
$$

(Note that this implies $\widetilde{P}(B, t) \leqq \mu(t)$ for all $B \in \mathscr{Z}$ ).

Proposition 3.3. Suppose that for some $K<\infty$,

$$
|\phi| \leqq K
$$

Then $E\left(L_{1}\right)=1$. 
Proof. From (3.28), (3.31) and (3.32),

$$
L_{t} \leqq(K+1) \exp K \mu(t) .
$$

Hence

$$
\left|L_{t^{-}} \phi(z, t)\right|^{2} \leqq K^{2}(K+1)^{2} \exp 2 K \mu(t)
$$

so that

$$
E \int_{Z} \int_{0}^{1}\left|L_{t^{-}} \phi(z, t)\right|^{2} \widetilde{P}(d z, d t) \leqq E \int_{0}^{1} K^{2}(K+1)^{2} \exp 2 K \mu(t) \mu(d t)<\infty,
$$

which implies that

$$
L_{t^{-}} \phi(z, t) \in L^{2}(\widetilde{P})
$$

By $\S 2.10, L_{t}$ is a square-integrable martingale, and in particular $E\left(L_{1}\right)=E\left(L_{0}\right)$ $=1$.

Proposition 3.4. Suppose that for some $K<\infty$,

$$
\int_{Z} \int_{0}^{1}(1+\phi(z, t))[\ln (1+\phi(z, t))]^{2} \widetilde{P}(d z, d t) \leqq K \quad \text { a.s. }
$$

Then $E\left(L_{1}\right)=1$.

Proof. Define the function $\phi^{n}$ so that

$$
\phi^{n}(z, \omega, t)= \begin{cases}\phi(z, \omega, t) & \text { if } 1 / n<1+\phi(z, \omega, t)<n, \\ 0 & \text { otherwise }\end{cases}
$$

and let $L_{t}^{n}$ be obtained from (3.28) by replacing $\phi$ with $\phi^{n}$. By Proposition 3.3, $E\left(L_{1}^{n}\right)=1$ and it is clear that $L_{1}^{n}$ converges to $L_{1}$ in probability. Hence by [3, $\S$ II, Thm. 21], $E\left(L_{1}\right)=1$ if and only if the set of r.v.s $\left\{L_{1}^{n} \mid n=1,2, \cdots\right\}$ is uniformly integrable. Define the probability measures $P_{n}$ by

$$
\frac{d P_{n}}{d P}(\omega)=L_{1}^{n}(\omega)
$$

By Theorem 3.3, $\left(x_{t}, \mathscr{F}_{t}, P_{n}\right), t \in[0,1]$, is a fundamental process with 1.d. $\left(\left(1+\phi^{n}\right) n, \Lambda\right)$ and so the corresponding margingales are given by

$$
Q_{n}(B, t)=P(B, t)-\widetilde{P}_{n}(B, t)=P(B, t)-\int_{B} \int_{0}^{t}\left(1+\phi^{n}(z, s)\right) \widetilde{P}(d z, d s) .
$$

Because $\phi^{n}$ is bounded and because of (3.31), $Q_{n} \in \mathscr{M}^{2}\left(P_{n}\right)$. For later reference define $\xi_{t}^{n} \in \mathscr{M}^{2}\left(P_{n}\right)$ by

$$
\xi_{t}^{n}=\left(\ln \left(1+\phi^{n}\right) \circ Q_{n}\right)_{t}
$$

and note that

$$
\left\langle\xi^{n}, \xi^{n}\right\rangle_{t}=\int_{Z} \int_{0}^{t}\left[\ln \left(1+\phi^{n}\right)\right]^{2}\left(1+\phi^{n}\right) \tilde{P}(d z, d s) .
$$


We are ready to show that $\left\{L_{1}^{n}\right\}$ is a uniformly integrable family. Fix $M<\infty$. First,

$$
\int_{\left\{L_{1}^{n}>M\right\}} L_{1}^{n}(\omega) P(d \omega)=P_{n}\left\{L_{1}^{n}>M\right\}
$$

Next,

$$
\begin{aligned}
\left\{L_{1}^{n}>\right. & M\} \\
= & \left\{\exp \left[\int_{Z} \int_{0}^{1} \ln \left(1+\phi^{n}\right) P(d z, d s)\right] \cdot \exp \left[-\int_{Z} \int_{0}^{1} \phi^{n} \tilde{P}(d z, d s)\right]>M\right\} \\
= & \left\{\exp \left[\int_{Z} \int_{0}^{1} \ln \left(1+\phi^{n}\right)\left[P(d z, d s)-\left(1+\phi^{n}\right) \tilde{P}(d z, d s)\right]\right]\right. \\
& \left.\cdot \exp \left[\int_{Z} \int_{0}^{1}\left[\left(1+\phi^{n}\right) \cdot \ln \left(1+\phi^{n}\right)-\phi^{n}\right] \tilde{P}(d z, d s)\right]>M\right\} \\
& \left\{\int_{Z} \int_{0}^{1} \ln \left(1+\phi^{n}\right)\left[P(d z, d s)-\left(1+\phi^{n}\right) \tilde{P}(d z, d s)\right]>\frac{1}{2} \ln M\right\} \\
& \cup\left\{\int_{Z} \int_{0}^{1}\left[\left(1+\phi^{n}\right) \ln \left(1+\phi^{n}\right)-\phi^{n}\right] \tilde{P}(d z, d s)>\frac{1}{2} \ln M\right\} \\
= & F_{1} \cup F_{2} \quad \text { say. }
\end{aligned}
$$

So

$$
P_{n}\left\{L_{1}^{n}>M\right\} \leqq P_{n}\left(F_{1}\right)+P_{n}\left(F_{2}\right) .
$$

From (3.33) it is immediate that $P_{n}\left(F_{2}\right)=0$ for all sufficiently large $M$. On the other hand, $F_{2}=\left\{\xi^{n}>\frac{1}{2} \ln M\right\}$, so by the Chebychev inequality,

$$
\begin{aligned}
P_{n}\left(F_{2}\right) & \leqq \frac{4}{(\ln M)^{2}} \int_{\Omega}\left\langle\xi^{n}, \xi^{n}\right\rangle_{1} d P_{n} \\
& \leqq \frac{4}{(\ln M)^{2}} K \quad(\text { by }(3.34),(3.33))
\end{aligned}
$$

It follows that for all $n, P_{n}\left\{L_{1}^{n}>M\right\} \rightarrow 0$ as $M \rightarrow \infty$, i.e., $\left\{L^{n}\right\}$ is uniformly integrable.

For the next proposition express $\widetilde{P}(d z, d s)=n(d z, s) \widetilde{P}(Z, d s)$ (see $\S 2.11)$.

Proposition 3.5. Suppose that there exist $\alpha>1$ and $K, K^{\prime}$ finite such that

$$
\int_{Z}(1+\phi(z, t))^{\alpha} n(d z, t) \leqq K+K^{\prime}[P(Z, t)+\widetilde{P}(Z, t)] \quad \text { a.s. }
$$

and suppose that for all $0<M<\infty$,

$$
E \exp [M P(Z, 1)]<\infty .
$$


Then for $1<\gamma \leqq \alpha^{1 / 2}$,

$$
\sup _{t \in[0,1]} E L_{t}^{\gamma}<\infty
$$

in particular $E L_{1}=1$.

Proof. If (3.37) is satisfied then by [8, $\S \mathrm{II}, \mathrm{Thm} .22]$ the family in $\left\{L_{t}\right.$; $0 \leqq t \leqq 1\}$ is uniformly integrable and so by $[8, \S \mathrm{VI}$, Thm. 6$], L_{t}$ is a uniformly integrable martingale, hence $E\left(L_{1}\right)=1$.

For $\alpha^{1 / 2} \geqq \gamma>1$, define

$$
\begin{aligned}
f_{t}(\gamma)= & \exp \left[\gamma \int_{Z} \int_{0}^{t} \ln (1+\phi) Q(d z, d s)\right. \\
& \left.+\int_{Z} \int_{0}^{t}\left[\gamma \ln (1+\phi)+\frac{1}{\gamma}-\frac{(1+\phi)^{\gamma^{2}}}{\gamma}\right] \tilde{P}(d z, d s)\right], \\
g_{t}(\gamma)= & \exp \left[\int_{Z} \int_{0}^{t}\left[-\gamma \phi-\frac{1}{\gamma}+\frac{(1+\phi)^{\gamma^{2}}}{\gamma}\right] \tilde{P}(d z, d s)\right]
\end{aligned}
$$

First of all,

$$
\begin{aligned}
f_{t}(\gamma) g_{t}(\gamma) & =\exp \left[\gamma \int_{Z} \int_{0}^{t} \ln (1+\phi) Q(d z, d s)-\int_{Z} \int_{0}^{t} \gamma(\phi-\ln (1+\phi)) \widetilde{P}(d z, d s)\right] \\
& =L_{t}^{\gamma} \quad(\text { by }(3.28)) .
\end{aligned}
$$

Next it can be checked by substitution in (3.28) that $\left[f_{t}(\gamma)\right]^{\gamma}$ is obtained from (3.28) by replacing $(1+\phi)$ with $(1+\phi)^{\gamma^{2}}$. Hence if $\gamma^{2}<2$ so that $(1+\phi)^{\gamma^{2}}$ $\in L_{\text {loc }}^{1}(\tilde{P})$, then we must have

$$
E\left[f_{t}(\gamma)\right]^{\gamma} \leqq 1 \text { for all } t
$$

Now by Hölder's inequality,

$$
E L_{t}^{\gamma} \leqq\left(E\left[f_{t}(\gamma)\right]^{\gamma}\right)^{1 / \gamma}\left(E\left[g_{t}(\gamma)\right]^{\gamma /(\gamma-1)}\right)^{(\gamma-1) / \gamma},
$$

so that

$$
E L_{t}^{\gamma} \leqq\left(E\left[g_{t}(\gamma)\right]^{\gamma /(\gamma-1)}\right)^{(\gamma-1) / \gamma}
$$

Next,

$$
\begin{aligned}
{\left[g_{t}(\gamma)\right]^{\gamma /(\gamma-1)} } & \leqq \exp \int_{Z} \int_{0}^{t}\left[\gamma-\frac{1}{\gamma}+\frac{(1+\phi)^{\gamma^{2}}}{\gamma}\right] \tilde{P}(d z, d s) \\
& \leqq \exp \left[\frac{\gamma^{2}-1}{\gamma} \mu(t)+\int_{0}^{t}\left\{K+K^{\prime}(P(Z, s)+\widetilde{P}(Z, s))\right\} \tilde{P}(Z, d s)\right] \\
& \leqq \exp \left[\left(\frac{\gamma^{2}-1}{\gamma}+K+\frac{K^{\prime}}{2} \mu(t)+K^{\prime} P(Z, t)\right) \mu(t)\right] \quad \text { (from (3.31), (3.35)) } \\
& \leqq \exp \beta \exp K^{\prime} \mu(1) P(Z, 1) \quad(\text { for some constant } \beta) .
\end{aligned}
$$


Hence,

$$
E L_{t}^{\gamma} \leqq(\exp \beta) E\left[\exp K^{\prime} \mu(1) P(Z, 1)\right]
$$

and the result follows from (3.36).

Remark 3.6. (i) Suppose $\left(x_{t}, \mathscr{F}_{t}, P\right)$ is as in the fundamental example with corresponding increasing processes $\tilde{p}_{1}(t), \cdots, \tilde{p}_{n}(t)$. Then Assumption 3.1 translates into the following: there exists an increasing function $\Lambda: R_{+} \rightarrow R_{+}$such that

$$
\sum_{i=1}^{n} \tilde{P}_{i}(t) \leqq \Lambda(t) \text { a.s. }
$$

Similarly (3.35), (3.36) become: there exist $\alpha>1$ and $K, K^{\prime}$ such that

$$
\sum_{i=1}^{n}\left(1+\phi_{i}(t)\right)^{\alpha} \leqq K+K^{\prime} \sum_{i=1}^{n}\left(p_{i}(t)+\tilde{p}_{i}(t)\right) .
$$

(ii) Now suppose that $\left(x_{t}, \mathscr{F}_{t}, P\right)$ is a standard Poisson process. Then (3.38) becomes

$$
(1+\phi(t))^{\alpha} \leqq K+K^{\prime}(x(t)+t)
$$

Suppose that $\phi(t)=c\left(x\left(t_{-}\right)\right)^{\alpha}$ for some $\alpha<1$. According to Feller [27, p. 452] a counting process $x_{t}$ with rate $[1+\phi(t)]$ has infinitely many jumps in a finite interval, so that it cannot be a fundamental process. Thus Proposition 3.5 is false if $\alpha<1$. We have been unable to resolve the case of "linear" growth, i.e., $\alpha=1$.

Remark 3.7. Propositions 3.3, 3.4, 3.5 are inspired by corresponding results in [6], [24], [28] respectively.

3.4. A class of Poisson-measure processes. In order to apply the transformation technique presented earlier one must begin with a fundamental process (with a known l.d.) whose existence is guaranteed. In this section we present a large class of such processes for which the increasing processes $\widetilde{P}(B, t)$ are deterministic.

Let $(Z, \mathscr{Z})$ be any Blackwell space and let $\mu$ be any positive measure on the space $\left(Z \times R_{+}, \mathscr{Z} \otimes \mathscr{B}\right)$, where $\mathscr{B}$ is the Borel field on $R_{+}$. Suppose that for all $t<\infty, \mu(Z \times[0, t])<\infty$.

Let $\Omega^{\prime}$ be the space of all (nonnegative) integer-valued measures $N$ on $\left(Z \times R_{+}, \mathscr{Z} \otimes \mathscr{B}\right)$. For each $T \in R_{+}$, let $\mathscr{F}_{T}^{\prime}$ be the family of all subsets of $\Omega^{\prime}$ of the form

$$
\left\{N \in \Omega^{\prime} \mid N(C) \in K\right\},
$$

where $C \in \mathscr{Z} \otimes \mathscr{B}[0, T]$ and $K \subset I_{+}$, the set of nonnegative integers. Evidently $\mathscr{F}^{\prime}$ is a $\sigma$-algebra on $\Omega^{\prime}$. Let

$$
\mathscr{F}^{\prime}=\bigvee_{T} \mathscr{F}_{T}^{\prime}
$$

Now, for each $T$ define the set function $P_{T}^{\prime}$ on $\left(\Omega^{\prime}, \mathscr{F}_{T}^{\prime}\right)$ by

$$
P_{T}^{\prime}(N(C) \in K)=\sum_{k \in K} \frac{\mu(C)^{k}}{k !} e^{-\mu(C)} .
$$


Note that $\mu(C)<\infty$ since $C \subset Z \times[0, T]$. By [31], $P_{T}^{\prime}$ defines a probability measure on $\left(\Omega^{\prime}, \mathscr{F}_{T}^{\prime}\right)$. Furthermore if $C_{1}, C_{2}$ are in $Z \times[0, T]$ and $C_{1} \cap C_{2}=\varnothing$, then the two random variables defined by

$$
N \mapsto N\left(C_{1}\right), \quad N \mapsto N\left(C_{2}\right), \quad N \in \Omega^{\prime},
$$

are independent. Finally the random variable $N \mapsto N(C)$ has a Poisson distribution. For $A \in \mathscr{Z}$, consider the counting process $P^{\prime}(A, t), t \in R_{+}$, defined on the family $\left(\Omega^{\prime}, \mathscr{F}_{t \wedge T}^{\prime}, P_{T}^{\prime}\right)$, by

$$
P^{\prime}(A, t)(N)=N(A \times[0, t \wedge T]) .
$$

Evidently $E\left(P^{\prime}(A, t)\right)=\mu(A \times[0, t \wedge T])$, and if $A_{1} \cap A_{2}=\varnothing$, then $P^{\prime}\left(A_{1}, t\right)$ and $P^{\prime}\left(A_{2}, t\right)$ are independent processes.

Next by Moyal [32], there exists a jump process $x_{t}, t \in R_{+}$, with values in $(Z, \mathscr{Z})$, defined on a family $\left(\Omega, \mathscr{F}_{t}^{x}, P_{T}\right)$ such that (i) $\left(\Omega, \mathscr{F}_{t}^{x}, P_{T}\right)$ is isomorphic to $\left(\Omega^{\prime}, \mathscr{F}_{t \wedge T}^{\prime}, P_{T}^{\prime}\right)$ and (ii) the counting processes $P^{x}(A, t)$ corresponding to $x_{t}$ are "isomorphic" to the processes $P^{\prime}(A, t)$ constructed above. Furthermore,

$$
\tilde{P}^{x}(A, t)=\mu(A \times[0, t \wedge T]) .
$$

To finish the construction we merely note that if $S<T$, then the probability measure $P_{S}$ on $\left(\Omega, \mathscr{F}_{S}^{x}\right)$ coincides with the restriction of $P_{T}$ (defined on $\mathscr{F}_{T}^{x}$ ) to $\mathscr{F}_{S}^{x}$. By the Kolmogorov consistency theorem, there therefore exists a probability measure $P$ on $\left(\Omega, \mathscr{F}_{\infty}^{x}\right)$ such that

$$
\tilde{P}^{x}(A, t)=\mu(A \times[0, t]), \quad A \in \mathscr{Z}, \quad t \in R_{+} .
$$

However the process $x_{t}$ may not be a fundamental process. To guarantee this we must be sure that the jump times are totally inaccessible. As mentioned in $\S 2.9$, this is equivalent to the requirement that $\widetilde{P}^{x}(A, t)$ have continuous sample paths, and hence, from (3.39), to the requirement that $\mu(A \times[0, t])$ be continuous in $t$ for each fixed $A$. We summarize the main conclusions as follows.

THEOREM 3.5. Let $(Z, \mathscr{Z})$ be a Blackwell space and let $\mu$ be any nonnegative measure on $\left(Z \times R_{+}, \mathscr{Z} \otimes \mathscr{B}\right)$ such that

(i) $\mu(Z \times[0, t])<\infty$ for all $t \in R_{+}$,

(ii) $\mu(A \times[0, t])$ is continuous in $t$ for all $A \in \mathscr{Z}$.

Then there exists a fundamental process $x_{t}$ on a family $\left(\Omega, \mathscr{F}_{t}^{x}, P\right)$ with values in $(Z, \mathscr{Z})$ such that

$$
\tilde{P}^{x}(A, t)=\mu(A \times[0, t]), \quad A \in \mathscr{Z}, \quad t \in R_{+} .
$$

Remark 3.8. (i) The $x_{t}$ process has independent increments in the sense that the $P(A, t)$ have independent increments. If $x_{t}$ were vector-valued this would indeed imply that $x_{t}$ has independent increments in the usual sense.

(ii) The most useful version of this result would be when $\mu$ is a product measure $\mu(d z, d s)=n(d z) \Lambda(d s)$, where $n$ is a finite measure on $(Z, \mathscr{Z})$ and $\Lambda(t)$ is a continuous increasing function on $R_{+}$, in which case $(n, \Lambda)$ would be a Lévy system.

4. Detection. The prototypical detection problem in communication theory is the following. We observe a sample $x_{t}(\omega), 0 \leqq t<\infty$, of a stochastic process. 
The process is known to be governed by one of two laws, $P$ or $P_{1}$. Based upon the observed sample one has to decide which of the two hypotheses, $P$ or $P_{1}$, is true. The term "detection" arises from a particular instance of this hypothesis testing model, namely, when the process $x$ has the representation

$$
\begin{array}{ll}
d x_{t}=\text { white noise }, & \text { under } P, \\
d x_{t}=\text { white noise }+s_{t}, & \text { under } P_{1},
\end{array}
$$

where $s_{t}$ is called the "signal". Thus deciding which hypothesis is true is, for the example, equivalent to "detecting" whether the signal is present (hypothesis $P_{1}$ ) or absent (hypothesis $P$ ).

Very recently this problem has been considered for the case where $x_{t}$ is a counting process under $P_{1}$ and a Poisson process under $P$ [6], [7], [15], [16], [17]. The case where $x_{t}$ is a Markov chain under $P$ has also been discussed [6]. We generalize these results by considering problems where $x_{t}$ is a fundamental process.

A well-established procedure for judging which hypothesis is true consists in first calculating the "likelihood" ratio $\left(d P_{1} / d P\right)(x(\omega))$ and then in accepting $P_{1}$ if $d P_{1} / d P>\alpha$ and rejecting $P_{1}$ otherwise. The selection of the "threshold" $\alpha$ is discussed in [18]. The procedure is often called the "threshold detector".

Evidently for this procedure to be meaningful one must assume $P_{1} \ll P$. Also to obtain results of practical value one must specify precisely how the "signal" affects the observation, as for instance in (4.1), where it is assumed to be additive. We proceed to the mathematical model.

Let $\left(\Omega, \mathscr{F}_{t}\right), t \in R_{+}$, be a family of spaces and $P, P_{1}$ two probabilities on $(\Omega, \mathscr{F})$. The observed process is a family of measurable functions $x_{t}:\left(\Omega, \mathscr{F}_{t}\right) \rightarrow(Z, \mathscr{Z})$ such that $\left(x_{t}, \mathscr{F}_{t}, P\right)$ and $\left(x_{t}, \mathscr{F}_{t}, P_{1}\right)$ are both fundamental processes. The processes $P, \widetilde{P}, Q$ and $\widetilde{P}^{x}, Q^{x}$ are the extrinsic and intrinsic (i.e., relative to $\mathscr{F}_{t}^{x}$ ) processes corresponding to $\left(x_{t}, P\right)$. Similarly $P_{1}, \widetilde{P}_{1}, \widetilde{P}_{1}^{x}$ etc. correspond to $\left(x_{t}, P_{1}\right)$. The extrinsic and intrinsic 1.d.'s are $(n, \Lambda),\left(n^{x}, \Lambda^{x}\right)$ for $\left(x_{t}, P\right)$ and $\left(n_{1}, \Lambda_{1}\right),\left(n_{1}^{x}, \Lambda_{1}^{x}\right)$ for $\left(x_{t}, P_{1}\right)$. (4.1).

We now give the model corresponding to the "signal plus noise" model of

Assumption 4.1. There exist $\mathscr{F}_{t}^{x}$-predictable processes $\mu(B, \omega, t), B \in \mathscr{Z}$, and $\mathscr{F}_{t}$-predictable processes $g(z, \omega, s)$ and $g_{1}(z, \omega, s)$ such that $E|g(z, s)|<\infty$ and $E_{1}\left|g_{1}(z, s)\right|<\infty$ for all $z, s$, and

$$
\begin{aligned}
& \widetilde{P}(B, t)=n(B, t) \Lambda(t)=\int_{B} \int_{0}^{t} g(z, \omega, s) \mu(d z, \omega, d s), \\
& \widetilde{P}_{1}(B, t)=n_{1}(B, t) \Lambda_{1}(t)=\int_{B} \int_{0}^{t} g_{1}(z, \omega, s) \mu(d z, \omega, d s),
\end{aligned}
$$

where the integrals are Stieltjes integrals.

Interpretation. In communication theory terms we can say that the "jump rates" $P(B, t)$ are "modulated" by the signal through the functions $g, g_{1}$.

DEFINITION 4.1. Let $E\left(g(z, t) \mid \mathscr{F}_{t}^{x}\right)=\hat{g}(z, t)$ and $E_{1}\left(g_{1}(z, t) \mid \mathscr{F}_{t}^{x}\right)=\hat{g}_{1}(z, t)$. 
Proposition 4.1.

$$
\begin{array}{ll}
\tilde{P}^{x}(B, t)=\int_{B} \int_{0}^{t} \hat{g}(z, s) \mu(d z, d s) & \text { a.s. } \\
\tilde{P}_{1}^{x}(B, t)=\int_{B} \int_{0}^{t} \hat{\mathrm{g}}_{1}(z, s) \mu(d z, d s) & \text { a.s. }
\end{array}
$$

Proof. It is enough to prove the first assertion since the proof for the second is identical. Fix $B \in \mathscr{Z}$. We know that

$$
\begin{aligned}
Q(B, t) & =P(B, t)-\int_{B} \int_{0}^{t} g(z, s) \mu(d z, d s) \in \mathscr{M}_{\mathrm{loc}}^{2}\left(\mathscr{F}_{t}, P\right) \\
Q^{x}(B, t) & =P(B, t)-\tilde{P}^{x}(B, t) \quad \in \mathscr{M}_{\mathrm{loc}}^{2}\left(\mathscr{F}_{t}^{x}, P\right) .
\end{aligned}
$$

Let $T_{n}, n=0,1, \cdots$, be the jump times of $x_{t}$. The $T_{n}$ are stopping times for the family $\left(\mathscr{F}_{t}\right)$ as well as for $\left(\mathscr{F}_{t}^{x}\right)$. Furthermore $E\left|P\left(B, t \wedge T_{n}\right)\right| \leqq n$. Hence

$$
E\left|Q\left(B, t \wedge T_{n}\right)\right|<\infty,
$$

and we can define a process $\left(\hat{Q}(B, t), \mathscr{F}_{t}^{x}, P\right)$ such that

$$
\hat{Q}\left(B, t \wedge T_{n}\right)=E\left(Q\left(B, t \wedge T_{n}\right) \mid \mathscr{F}_{t}^{x}\right)
$$

and it is trivial that $\hat{Q}\left(B, t \wedge T_{n}\right) \in \mathscr{M}^{1}\left(\mathscr{F}_{t}^{x}, P\right)$. Now $P(B, t)$ and $\mu(z, t)$ are $\mathscr{F}_{t}^{x}-$ measurable, hence

$$
\hat{Q}\left(B, t \wedge T_{n}\right)=P\left(B, t \wedge T_{n}\right)-\int_{B} \int_{0}^{t \wedge T_{n}} E\left(g(z, s) \mid \mathscr{F}_{t}^{x}\right) \mu(d z, d s) .
$$

Subtracting this from (4.3) implies that

$$
\tilde{P}^{x}\left(B, t \wedge T_{n}\right)-\int_{B} \int_{0}^{t \wedge T_{n}} E\left(g(z, s) \mid \mathscr{F}_{t}^{x}\right) \mu(d z, d s) \in \mathscr{M}^{1}\left(\mathscr{F}_{t}^{x}, P\right) .
$$

On the other hand it can be directly verified that

$$
\int_{B} \int_{0}^{t \wedge T_{n}}\left[E\left(g(z, s) \mid \mathscr{F}_{t}^{x}\right)-\hat{g}(z, s)\right] \mu(d z, d s) \in \mathscr{M}^{1}\left(\mathscr{F}_{t}^{x}, P\right) .
$$

Therefore

$$
\tilde{P}^{x}\left(B, t \wedge T_{n}\right)-\int_{B} \int_{0}^{t \wedge} \hat{g}(z, s) \mu(d z, d s) \in \mathscr{M}^{1}\left(\mathscr{F}_{t}^{x}, P\right) .
$$

But this is a continuous process. Hence it must vanish, i.e.,

$$
\widetilde{P}^{x}(B,)=\int_{B} \int_{0}^{t} \hat{g}(z, s) \mu(d z, d s) .
$$

Remark 4.1. The processes $\left(\hat{Q}(B, t), \mathscr{F}_{t}^{x}, P\right)$ are called the innovations processes of the process $\left(x_{t}, \mathscr{F}_{t}, P\right)$, in analogy with the Brownian motion case [21]. These processes will be used in the next section. 
Theorem 4.1. Suppose that $P_{1} \ll P$. Let $L_{t}=E\left(d P_{1} / d P \mid \mathscr{F}_{t}^{x}\right)$ be the likelihood ratio and let

$$
T=\inf \left\{t \mid L_{t}=0 \text { or } L_{t^{-}}=0\right\} .
$$

Then there is a sequence of $\mathscr{F}_{t}^{x}$ s.t.'s $S_{k} \uparrow T$ a.s. $P$ such that

$$
\frac{\hat{\mathrm{g}}_{1}(z, s)}{\hat{\mathrm{g}}(z, s)} I_{\left\{s \leqq S_{k}\right\}} \in L_{\mathrm{loc}}^{1}\left(\tilde{P}^{x}\right)
$$

and

$$
L_{t \wedge S_{k}}=\prod_{\substack{s \leqq t \wedge S_{k} \\ x_{s^{-}} \neq x_{s}}}\left[\frac{\hat{g}_{1}\left(x_{s}, s\right)}{\hat{g}\left(x_{s}, s\right)}\right] \exp \left[-\int_{Z} \int_{0}^{t \wedge S_{k}}\left(\frac{\hat{g}_{1}(z, s)}{\hat{g}(z, s)}-1\right) \hat{g}(z, s) \mu(d z, d s)\right]
$$

Proof. By Theorem 3.1 there exists s.t.'s $S_{k} \uparrow T$ and an $\mathscr{F}_{t}^{x}$-predictable function $\phi$ such that $L_{t \wedge S_{k}}$ is given by (3.11), and by Theorem 3.2 the intrinsic 1.d. of $\left(x_{t}, P_{1}\right)$ is $\left((1+\phi) n^{x}, \Lambda^{x}\right)$, where $\left(n^{x}, \Lambda^{x}\right)$ is the intrinsic 1.d. of $\left(x_{t}, P\right)$; so from Proposition 4.1 we can conclude that

$$
\begin{aligned}
(1+\phi(z, s)) n^{x}(d z, s) \Lambda^{x}(d s) & =(1+\phi(z, s)) \hat{g}(z, s) \mu(d z, d s) \\
& =\hat{g}_{1}(z, s) \mu(d z, d s)=n_{1}^{x}(d z, s) \Lambda_{1}^{x}(d s) .
\end{aligned}
$$

Therefore

$$
1+\phi(z, s)=\frac{\hat{g}_{1}(z, s)}{\hat{g}(z, s)}
$$

which upon substitution into (3.11) yields (4.4).

COROllary 4.1. Suppose in the above that $x_{t}$ is as in the fundamental example of $\S 2.12$. Suppose there exists a $\mathscr{F}_{t}^{x}$-predictable process $\mu(t)$, and $\mathscr{F}_{t}$-predictable processes $\lambda^{i}(t), \lambda_{1}^{i}(t), 1 \leqq i \leqq n$, such that

$$
\tilde{p}_{i}(t)=\int_{0}^{t} \lambda^{i}(s) \mu(d s), \quad \tilde{p}_{i, 1}(t)=\int_{0}^{t} \lambda_{1}^{i}(s) \mu(d s), \quad 1 \leqq i \leqq n .
$$

Then the formula (4.4) changes to

$$
L_{t \wedge S_{k}}=\prod_{i=1}^{n}\left\{\prod_{\substack{s \leqq t \wedge S_{k} \\\left(x_{s}-, x_{s}\right) \in \sigma_{i}}}\left[\frac{\hat{\lambda}_{1}^{i}(s)}{\hat{\lambda}^{i}(s)}\right] \cdot \exp \left[-\int_{0}^{t \wedge S_{k}}\left(\frac{\hat{\lambda}_{1}^{i}(s)}{\hat{\lambda}^{i}(s)}-1\right) \hat{\lambda}^{i}(s) \mu(d s)\right]\right\} .
$$

Proof. The proof follows from Theorem 4.1 and Remark 3.2 (iii).

Remark 4.2. (i) Very special cases of (4.5) have appeared in the recent literature. Suppose in Corollary 4.1 that $\left(x_{t}, \mathscr{F}_{t}^{x}, P\right)$ is a Poisson process with rate $\lambda_{0}$. Then in (4.5), $n=1, \hat{\lambda}(s) \equiv \lambda_{0}, \mu(d s) \equiv d s$ and (4.5) becomes

$$
L_{t \wedge s_{k}}=\left[\prod_{\substack{s \leqq t \wedge s_{k} \\ x_{s-} \neq x_{s}}} \frac{\hat{\lambda}_{0}(s)}{\lambda_{0}}\right] \exp \left[-\int_{0}^{t \wedge s_{k}}\left(\hat{\lambda}_{1}(s)-\lambda_{0}\right) d s\right] \text {. }
$$

This version together with the comment in footnote 5 yields the result in $[16$, p. 95]. Actually in [16] some strong unnecessary assumptions are also imposed. 
Formula (4.6) has also been derived in [6] and [7]. Formula (4.5) for the case $n=1$ and $\mu(d s) \equiv d s$ appears in [15], although the derivation is not satisfactory, and various additional assumptions, some of which are not easily unverifiable, were made there.

(ii) In [6] we can also find (4.5) for the special case where $\left(x_{t}, \mathscr{F}_{t}^{x}, P\right)$ is a Markov chain, in which case the $\hat{\lambda}^{i}$ can be interpreted in terms of various transition probabilities as suggested in $\S 2.11, \S 2.12$.

We apply formulas (4.5) and (4.6) to calculate the mutual information between two fundamental processes. Let $x_{t}$ and $x_{t}^{\prime}$ be two such processes on $\left(\Omega, \mathscr{F}_{t}, P\right)$ with values in $(Z, \mathscr{Z})$ and $\left(Z^{\prime}, \mathscr{Z}^{\prime}\right)$ respectively. Let $\mu(d z, d s)$ and $\mu^{\prime}\left(d z^{\prime}, d s\right)$ be $\mathscr{F}_{t}^{x}$ - and $\mathscr{F}_{t}^{x^{\prime}}$-predictable processes and $g(z, s), g^{\prime}\left(z^{\prime}, s\right)$ be two $\mathscr{F}_{t}$-predictable processes with finite expectation such that

$$
\begin{aligned}
n(d z, s) \Lambda(d s) & =g(z, s) \mu(d z, d s), \\
n^{\prime}\left(d z^{\prime}, s\right) \Lambda^{\prime}(d s) & =g^{\prime}\left(z^{\prime}, s\right) \mu^{\prime}\left(d z^{\prime}, d s\right) .
\end{aligned}
$$

Let $P_{x}, P_{x^{\prime}}$ denote the restrictions of $P$ to $\mathscr{F}^{x}$ and $\mathscr{F}^{x^{\prime}}$ respectively. Assume that $\mathscr{F}_{t}=\mathscr{F}_{t}^{x} \otimes \mathscr{F}_{t}^{x^{\prime}}$, the product $\sigma$-algebra and let $P_{x x^{\prime}}=P_{x} \otimes P_{x^{\prime}}$ denote the product measure on $\mathscr{F}=\mathscr{F}^{x} \otimes \mathscr{F}^{x^{\prime}}$. It is trivial that $P \ll P_{x x^{\prime}}$. Assume further that $P_{x x^{\prime}} \ll P$. The mutual information between $x, x^{\prime}$ is the quantity

$$
I\left(x, x^{\prime}\right)=E\left(\ln \frac{d P}{d P_{x x^{\prime}}}\right) .
$$

Let

$$
\begin{aligned}
\hat{g}(z, t) & =E\left(g(z, t) \mid \mathscr{F}_{t}^{x}\right), \\
\hat{g}^{\prime}\left(z^{\prime}, t\right) & =E\left(g^{\prime}\left(z^{\prime}, t\right) \mid \mathscr{F}_{t}^{x^{\prime}}\right) .
\end{aligned}
$$

By Remark 3.2 (i),

$$
g / \hat{g} \in L_{\text {loc }}^{1}(\widetilde{P}), \quad g^{\prime} / \hat{g}^{\prime} \in L_{\text {loc }}^{1}\left(\widetilde{P}^{\prime}\right) .
$$

Assume further that

$$
\ln (g / \hat{g}) \in L^{1}(\widetilde{P}), \quad \ln \left(g^{\prime} / \hat{g}^{\prime}\right) \in L^{1}\left(\tilde{P}^{\prime}\right) .
$$

Then by Theorem 4.1,

$$
\begin{aligned}
\frac{d P}{d P_{x x^{\prime}}}= & \left\{\prod_{x_{s} \neq x_{s}}\left[\frac{g\left(x_{s}, s\right)}{\hat{g}\left(x_{s}, s\right)}\right] \exp \left[-\int_{Z} \int_{0}^{\infty}\left(\frac{g(z, s)}{\hat{g}(z, s)}-1\right) \hat{g}(z, s) \mu(d z, d s)\right]\right\} \\
& \cdot\left\{\prod_{x_{s^{\prime}} \neq x_{s}^{\prime}}\left[\frac{g^{\prime}\left(x_{s}^{\prime}, s\right)}{\hat{g}^{\prime}\left(x_{s}^{\prime}, s\right)}\right] \exp \left[-\int_{Z^{\prime}} \int_{0}^{\infty}\left(\frac{g^{\prime}\left(z^{\prime}, s\right)}{\hat{g}^{\prime}\left(z^{\prime}, s\right)}-1\right) \hat{g}^{\prime}(z, s) \mu^{\prime}\left(d z^{\prime}, d s\right)\right]\right\}
\end{aligned}
$$

so that

$$
\begin{aligned}
\ln \frac{d P}{d P_{x x^{\prime}}}= & \sum_{x_{s}-\neq x_{s}} \ln \left(\frac{g\left(x_{s}, s\right)}{\hat{g}\left(x_{s}, s\right)}\right)-\int_{Z} \int_{0}^{\infty}\left(\frac{g(z, s)}{\hat{g}(z, s)}-1\right) \hat{g}(z, s) \mu(d z, d s) \\
& +\sum_{x_{s}^{\prime} \neq x_{s}} \ln \left(\frac{g^{\prime}\left(x_{s}^{\prime}, s\right)}{\hat{g}^{\prime}\left(x_{s}^{\prime}, s\right)}\right)-\int_{Z^{\prime}} \int_{0}^{\infty}\left(\frac{g^{\prime}\left(z^{\prime}, s\right)}{\hat{g}^{\prime}\left(z^{\prime}, s\right)}-1\right) \hat{g}^{\prime}\left(d z^{\prime}, d s\right) \mu^{\prime}\left(d z^{\prime}, d s\right) .
\end{aligned}
$$


Since $\ln (g / \hat{g}) \in L^{1}(\widetilde{P})$, therefore

$$
\begin{aligned}
\sum_{x_{s^{-}} \neq x_{s}} \ln \left(\frac{g\left(x_{s}, s\right)}{\hat{g}\left(x_{s}, s\right)}\right)-\int_{Z} \int_{0}^{\infty} \ln \left\langle\frac{g(z, s)}{\hat{g}(z, s)}\right) g(z, s) \mu(d z, d s) \\
=\int_{Z} \int_{0}^{\infty} \ln \left(\frac{g(z, s)}{\hat{g}(z, s)}\right)[P(d z, d s)-\tilde{P}(d z, d s)] \in \mathscr{M}^{1}\left(\mathscr{F}_{t}, P\right)
\end{aligned}
$$

so that

$$
E\left[\sum_{x_{s^{-}} \neq x_{s}} \ln \left(\frac{g\left(x_{s}, s\right)}{\hat{g}\left(x_{s}, s\right)}\right)\right]=E \int_{Z} \int_{0}^{\infty} \ln \left(\frac{g(z, s)}{\hat{g}(z, s)}\right) g(z, s) \mu(d z, d s) .
$$

Similarly,

$$
E\left[\sum_{x_{s}^{\prime} \neq x_{s}} \ln \left(\frac{g^{\prime}\left(x_{s}^{\prime}, s\right)}{\hat{g}^{\prime}\left(x_{s}^{\prime}, s\right)}\right)\right]=E \int_{Z^{\prime}} \int_{0}^{\infty} \ln \left(\frac{g^{\prime}\left(z^{\prime}, s\right)}{\hat{g}^{\prime}\left(z^{\prime}, s\right)}\right) g^{\prime}\left(z^{\prime}, s\right) \mu^{\prime}\left(d z^{\prime}, d s\right) .
$$

Taking expectations in (4.7) and substituting these relations gives the following result.

THEOREM 4.2. Suppose $P_{x x^{\prime}} \ll P$ and $\ln (g / \hat{g}) \in L^{1}(\widetilde{P}), \ln \left(g^{\prime} / \hat{g}^{\prime}\right) \in L^{1}(\widetilde{P})$. Then

$$
\begin{aligned}
I\left(x, x^{\prime}\right)= & E\left[\int_{Z} \int_{0}^{\infty}\left(\ln \frac{g(z, s)}{\hat{g}(z, s)}+\frac{\hat{g}(z, s)}{g(z, s)}-1\right) g(z, s) \mu(d z, d s)\right. \\
& \left.+\int_{Z^{\prime}} \int_{0}^{\infty}\left(\ln \frac{g^{\prime}\left(z^{\prime}, s\right)}{\hat{g}^{\prime}\left(z^{\prime}, s\right)}+\frac{\hat{g}^{\prime}\left(z^{\prime}, s\right)}{g^{\prime}\left(z^{\prime}, s\right)}-1\right) g^{\prime}\left(z^{\prime}, s\right) \mu^{\prime}\left(d z^{\prime}, d s\right)\right] .
\end{aligned}
$$

Remark 4.3. This result for the case where $x, x^{\prime}$ are both counting processes has appeared in [6], and our proof is adapted from the one given there.

5. Filtering. A popular model for estimation and filtering problems in communication and control is where the observed process, $x_{t}$, depends upon the "signal" or "state" process, $y_{t}$, according to

$$
\begin{aligned}
& d y_{t}=g\left(y_{t}\right) d t+d B_{1}(t), \\
& d x_{t}=f\left(x_{t}, y_{t}\right) d t+d B_{2}(t),
\end{aligned}
$$

where $B_{1}, B_{2}$ are Brownian motions. The problem is to determine $E\left(y_{t} \mid \mathscr{F}_{t}^{x}\right)$. Note that in the above $y_{t}$ is a semi-martingale.

We begin this section by examining this situation when $\left(x_{t}, \mathscr{F}_{t}, P\right)$ is a fundamental process with values in $(Z, \mathscr{Z})$. We need a preliminary fact.

LEMMA 5.1. Let $\left(m_{t}, \mathscr{F}_{t}, P\right) \in \mathscr{M}^{2}\left(\mathscr{F}_{t}, P\right)$. Then there exists an $\mathscr{F}_{t}$-predictable process $h(z, t)$ such that

$$
E \int_{Z} \int_{0}^{\infty}|h(z, t)|^{2} \widetilde{P}(d z, d t)<\infty
$$

and

$$
\left\langle m_{t}, Q(B, t)\right\rangle=\int_{B} \int_{0}^{t} h(z, s) \widetilde{P}(d z, d s) \quad \text { for all } B \in \mathscr{Z} .
$$


Proof. The set, say $\mathscr{L}$, of all processes $(h \circ Q)_{t}$, where $h$ is any predictable process satisfying (5.1), is easily shown to be a stable subspace of $\mathscr{M}^{2}\left(\mathscr{F}_{t}, P\right)$ (see [19] for a definition of a stable subspace). Therefore by [19], there exists a unique decomposition of $m_{t}, m_{t}=n_{t}+l_{t}$, with $l_{t} \in \mathscr{L}$ and $\left\langle n_{t}, l_{t}^{\prime}\right\rangle \equiv 0$ for all $l_{t}^{\prime} \in \mathscr{L}$. Let $l_{t}=(h \circ Q)_{t}$ and the assertion follows.

Assumption 5.1. There exist $\mathscr{F}_{t}^{x}$-predictable processes $\mu(B, t), B \in \mathscr{Z}$, and an $\mathscr{F}_{t}$-predictable process $g(z, t)$ such that

$$
\widetilde{P}(B, t)=\int_{B} \int_{0}^{t} g(z, s) \mu(d z, d s) .
$$

Notation. In the following for any process $\left(f_{t}, \mathscr{F}_{t}, P\right), \hat{f}_{t}=E\left(f_{t} \mid \mathscr{F}_{t}^{x}\right)$.

THEOREM 5.1. Let $\left(x_{t}, \mathscr{F}_{t}, P\right)$ be a fundamental process satisfying Assumption 5.1. Let $\left(y_{t}, \mathscr{F}_{t}, P\right) \in \mathscr{S}\left(\mathscr{F}_{t}\right)$ have the representation

$$
y_{t}=y_{0}+a_{t}+m_{t}
$$

with $a_{t} \in \mathscr{A}\left(\mathscr{F}_{t}\right), m_{t} \in \mathscr{M}^{2}\left(\mathscr{F}_{t}\right)$. Then $\hat{y}_{t}$ satisfies the filtering equation

$$
\hat{y}_{t}=\hat{y}_{0}+\eta_{t}+\int_{z} \int_{0}^{t} k(z, s) Q^{x}(d z, d s)
$$

where $\eta_{t} \in \mathscr{A}\left(\mathscr{F}_{t}^{x}\right), Q^{x}(B, t)=P(B, t)-\int_{B} \int_{0}^{t} \hat{g}(z, s) \mu(d z, d s)$, and where the $\mathscr{F F}_{t}^{x}$ predictable process $k$ satisfies

$$
k(z, s)=\frac{\widehat{\left[\left(y_{s^{-}}-\hat{y}_{s^{-}}+h(z, s)\right] g(z, s)\right.}}{\hat{\mathrm{g}}(z, s)}
$$

and $h, g$ are as in (5.2), (5.3) respectively.

Proof. Let $\mu_{t}=E\left(m_{t} \mid \mathscr{F}_{t}^{x}\right)$. Clearly $\mu_{t} \in \mathscr{M}^{2}\left(\mathscr{F}_{t}^{x}\right)$. Now write $a_{t}=a_{t}^{+}-a_{t}^{-}$ where $a_{t}^{+}, a_{t}^{-} \in \mathscr{A}_{+}\left(\mathscr{F}_{t}, P\right)$. It is easy to verify that the $\mathscr{F}_{t}^{x}$-measurable processes $\alpha_{t}^{+}=E\left(a_{t}^{+} \mid \mathscr{F}_{t}^{x}\right), \alpha_{t}^{-}=E\left(a_{t}^{-} \mid \mathscr{F}_{t}^{x}\right)$ are submartingales. By the Doob-Meyer decomposition theorem [3], there exist martingales $\xi_{t}^{+}, \xi_{t}^{-}$in $\mathscr{M}^{1}\left(\mathscr{F}_{t}^{x}\right)$ and $\mathscr{F}_{t}^{x}$ predictable increasing processes $\eta_{t}^{+}, \eta_{t}^{-}$in $\mathscr{A}_{+}\left(\mathscr{F}_{t}^{x}\right)$ such that

Hence

$$
\alpha_{t}^{+}=\xi_{t}^{+}+\eta_{t}^{+}, \quad \alpha_{t}^{-}=\xi_{t}^{-}+\eta_{t}^{-} \text {. }
$$

$$
\begin{aligned}
\hat{y}_{t} & =\hat{y}_{0}+\alpha_{t}^{+}-\alpha_{t}^{-}+\hat{m}_{t} \\
& =\hat{y}_{0}+\left(\eta_{t}^{+}-\eta_{t}^{-}\right)+\left(\xi_{t}^{+}-\xi_{+}^{-}+\mu_{t}\right) \\
& =\hat{y}_{0}+\eta_{t}+\xi_{t}, \text { say, }
\end{aligned}
$$

where $\eta_{t} \in \mathscr{A}\left(\mathscr{F}_{t}^{x}\right), \xi_{t} \in \mathscr{M}^{1}\left(\mathscr{F}_{t}^{x}\right)$. By $\S 2.11$ there exists a $\mathscr{F}_{t}^{x}$-predictable process $k(z, s) \in L_{\text {loc }}^{1}\left(\widetilde{P}^{x}\right)$ such that

$$
\xi_{t}=\int_{Z} \int_{0}^{t} k(z, s) Q^{x}(d z, d s)
$$

It remains to evaluate $k$. By the differentiation formula of $\S 2.7$,

$$
y_{t} P(B, t)=\int_{0}^{t} y_{s^{-}} P(B, d s)+\int_{0}^{t} P\left(B, s^{-}\right) d y_{s}+\left[m_{t}, Q(B, t)\right] .
$$


Since $P(B, t)-\tilde{P}(B, t)$ and $\left[m_{t}, Q(B, t)\right]-\left\langle m_{t}, Q(B, t)\right\rangle$ are in $\mathscr{M}_{\text {loc }}^{1}\left(\mathscr{F}_{t}\right)$, therefore, from the above, for some $\gamma_{t}, \gamma_{t}^{\prime} \in \mathscr{M}_{\mathrm{loc}}^{1}\left(\mathscr{F}_{t}\right)$,

$$
\begin{aligned}
y_{t} P(B, t) & =\int_{0}^{t} y_{s^{-}} \tilde{P}(B, d s)+\int_{0}^{t} P\left(B, s^{-}\right) d y_{s}+\left\langle m_{t}, Q(B, t)\right\rangle+\gamma_{t} \\
& =\int_{B} \int_{0}^{t}\left(y_{s^{-}}+h(z, s)\right) g(z, s) \mu(d z, d s)+\int_{0}^{t} P\left(B, s^{-}\right) d a_{s}+\gamma_{t}^{\prime},
\end{aligned}
$$

using (5.2), (5.3) and (5.4).

Now apply the differential rule to $\hat{y}_{t} P(B, t)$ to obtain

$$
\hat{y}_{t} P(B, t)=\int_{0}^{t} \hat{y}_{s^{-}} P(B, d s)+\int_{0}^{t} P\left(B, s^{-}\right) d \hat{y}_{s}+\left[\xi_{t}, Q^{x}(B, t)\right] .
$$

Recalling that $P(B, t)-\widetilde{P}^{x}(B, t)$ and $\left[\xi_{t}, Q^{x}(B, t)\right]-\left\langle\xi_{t}, Q^{x}(B, t)\right\rangle$ are in $\mathscr{M}_{\text {loc }}^{1}\left(\mathscr{F}_{t}^{x}\right)$, the relation above implies that for some $\delta_{t}, \delta_{t}^{\prime} \in \mathscr{M}_{\mathrm{loc}}^{1}\left(\mathscr{F}_{t}^{x}\right)$,

$$
\begin{aligned}
\hat{y}_{t} P(B, t) & =\int_{0}^{t} \hat{y}_{s^{-}} \tilde{P}^{x}(B, d s)+\int_{0}^{t} P\left(B, s^{-}\right) d \hat{y}_{s}+\left\langle\xi_{t}, Q^{x}(B, t)\right\rangle+\delta_{t} \\
& =\int_{B} \int_{0}^{t}\left(\hat{y}_{s^{-}}+k(z, s)\right) \hat{g}(z, s) \mu(d z, d s)+\int_{0}^{t} P\left(B, s^{-}\right) d \eta_{s}+\delta_{t}^{\prime},
\end{aligned}
$$

using Proposition 4.1, (5.5), (5.6).

Next we make the following observations, which can be verified directly from the martingale definition:

$$
\begin{aligned}
& \left(\widehat{\int_{B} \int_{0}^{t}\left(y_{s^{-}}+h(z, s)\right) g(z, s) \mu(d z, d s)}\right) \\
& \quad-\int_{B} \int_{0}^{t}\left[\left(\widehat{\left.y_{s^{-}}+h(z, s)\right) g(z, s}\right)\right] \mu(d z, d s) \in \mathscr{M}_{\mathrm{loc}}^{1}\left(\mathscr{F}_{t}^{x}\right), \\
& \left(\widehat{\int_{0}^{t} P\left(B, s^{-}\right) d a_{s}}\right)-\int_{0}^{t} P\left(B, s^{-}\right) d \eta_{s} \in \mathscr{M}_{\mathrm{loc}}^{1}\left(\mathscr{F}_{t}^{x}\right) .
\end{aligned}
$$

Using these facts and the fact that $\left(\widehat{y_{t} P(B, t)}\right)=\hat{y}_{t} P(B, t)$, we conclude from (5.7), (5.8) that

$$
\int_{B} \int_{0}^{t}\left\{\left(\hat{y}_{s^{-}}+k(z, s)\right) \hat{g}(z, s)-\left[\left(\widehat{\left.y_{s^{-}}+h(z, s)\right) g(z, s)}\right)\right\} \mu(d z, d s) \in \mathscr{M}_{\mathrm{loc}}^{1}\left(\mathscr{F}_{t}^{x}\right),\right.
$$

and since this process is continuous, it must vanish identically, so that we may assume

$$
\begin{aligned}
k(z, s) & =\frac{\left[\left(\widehat{\left.y_{s^{-}}+h(z, s)\right) g(z, s)}\right]\right.}{\hat{g}(z, s)}-\hat{y}_{s^{-}} \\
& =\frac{\left[\left(\widehat{\left.y_{s^{-}}-\hat{y}_{s^{-}}+h(z, s)\right) g(z, s)}\right]\right.}{\hat{g}(z, s)} .
\end{aligned}
$$


Corollary 5.1. Suppose in the above that $x_{t}$ is as in the fundamental example of $\S 2.12$ and that there exists an $\mathscr{F}_{t}^{x}$-predictable process $\mu(t)$ and $\mathscr{F}_{t}$-predictable processes $\lambda_{t}^{i}$ such that

$$
\tilde{p}_{i}(t)=\int_{0}^{t} \lambda^{i}(s) \mu(d s)
$$

and let $\left\langle m_{t}, q_{i}(t)\right\rangle=\int_{0}^{t} h_{i}(s) \tilde{p}_{i}(d s)$ for some $\mathscr{F}_{t}$-predictable processes $h_{i}$. Then

$$
\hat{y}_{t}=\hat{y}_{0}+\eta_{t}+\sum_{i=1}^{n} \int_{0}^{t} k_{i}(s) q_{i}^{x}(d s)
$$

with

$$
k_{i}(t)=\frac{\left[\left(\overline{\left.\hat{y}_{t^{-}}-\hat{y}_{t^{-}}+h_{i}(t)\right) \lambda^{i}(t)}\right)\right]}{\hat{\lambda}^{i}(t)}, \quad 1 \leqq i \leqq n
$$

and

$$
q_{i}^{x}(t)=p_{i}(t)-\int_{0}^{t} \hat{\lambda}^{i}(s) d s .
$$

Remark 5.1. (i) Suppose in (5.4) that $a_{t}$ is given as

$$
a_{t}=\int_{0}^{t} \beta_{s} d s
$$

for some predictable process $\beta_{t}$ in $L^{1}\left(\mathscr{F}_{t}\right)$. Since $\hat{a}_{t}-\int_{0}^{t} \hat{\beta}_{s} d s$ is in $\mathscr{M}^{1}\left(\mathscr{F}_{t}^{x}\right)$ it follows that in the representation for $\hat{y}_{t}$ we have the further specification

$$
\eta_{t}=\int_{0}^{t} \hat{\beta}_{s} d s
$$

(ii) Corollary 5.1 has appeared in the literature for the case where $\left(x_{t}, \mathscr{F}_{t}, P\right)$ is a counting process, i.e., $n=1$. Even here some additional conditions have been imposed on the $y_{t}$ process (such as, e.g., $y_{t}$ is Markov [6], [16]) or on the $x_{t}$ process (such as, e.g., $\left(x_{t}, \underset{\mathscr{F}_{t}}{x}, P\right)$ is obtained from a Poisson process by an absolutely continuous change of measure [6], [20]).

(iii) Theorem 5.1 has been inspired largely by the procedures of [21], where the underlying process is Brownian motion. See also [24] for the Brownian motion case.

(iv) While Theorem 5.1 has some value in terms of clarifying the issues involved in obtaining the filtering equations it is of little practical importance since these equations do not lead to a realization by a dynamical system. This is so because the filtering equations contain the terms $\eta_{t}, k_{t}$ and $\hat{g}_{t}$ which are not computable in terms of $\hat{y}_{t}$ and $x_{t}$. In other words, the filtering equation is not recursive. This difficulty persists even when one imposes additional conditions such as $y_{t}$ is Markov. In the remainder of this section we seek to determine conditions under which the filter is recursive.

We impose conditions on the dependence between the "signal" or "state" process $y_{t}$ and the "observation" process $x_{t}$ which are considerably stronger than 
those of Assumption 5.1. For the remainder of this section the following assumption holds.

Assumption 5.2. $\left(\Omega, \mathscr{F}_{t}\right), t \in R_{+}$, is a family of spaces and $P, P_{1}$ are two probability measures on $(\Omega, \mathscr{F}) . x_{t}$ and $y_{t}$ are measurable functions on $\left(\Omega, \mathscr{F}_{t}\right)$ with values in $(Z, \mathscr{Z})$ and $(Y, \mathscr{Y})$ respectively. The following properties are satisfied.

(i) $Z$ is a Borel subset of $R^{n}, \mathscr{Z}$ is the Borel field. (The most important practical cases are $Z=R^{n}$ or $Z$ is the space of all $z \in R^{n}$ with integer components.) $Y$ is a locally compact Hausdorff space, $\mathscr{Y}$ is the Borel field. $\mathscr{F}_{t}=\mathscr{F}_{t}^{x} \vee \mathscr{F}_{t}^{x}$.

(ii) Under the measure $P$,

(a) $\left(x_{t}, \mathscr{F}_{t}, P\right)$ is a fundamental process with independent increments, i.e., $x_{t}-x_{s}$ is independent of $\mathscr{F}_{s}($ under $P$ ), for $s \leqq t$,

(b) $\left(y_{t}, \mathscr{F}_{t}, P\right)$ is a Markov process whose sample paths are rightcontinuous and have left-limits, and the jump times of $y$ are totally inaccessible, dependent.

(c) the processes $x_{t}$ and $y_{t}$ are independent, i.e., $\mathscr{F}^{x}$ and $\mathscr{F}^{y}$ are intation

(iii) $P_{1} \ll P$, there exists an $\mathscr{F}_{t}$-predictable process $f \in L_{\text {loc }}^{1}(\widetilde{P})$ with a represen-

$$
f(z, \omega, t)=\phi\left(z, y_{t^{-}}(\omega), \omega, t\right),
$$

where $\phi(\cdot, y, \cdot, \cdot)$ is $\mathscr{F F}_{t}^{x}$-predictable for fixed $y \in Y$, and there also exist $\mathscr{F}_{t}{ }^{-}$ predictable processes $\mu(B, t)$ for $B \in \mathscr{Z}$ such that $E(|f(z, t)|)+E_{1}(|f(z, t)|)<\infty$ for all $z, t$ and

$$
L_{t}=E\left(\frac{d P_{1}}{d P} \mid \mathscr{F}_{t}\right)=\prod_{\substack{s \leqq t \\ s_{s^{-}} \neq x_{s}}}\left[1+\phi\left(x_{s}, y_{s^{-}}, s\right)\right] \exp \left[-\int_{Z} \int_{0}^{t} \phi\left(z, y_{s^{-}}, s\right) \mu(d z, d s)\right] .
$$

Note that we must have $1+\phi \geqq 0$.

Let $Q, \widetilde{P}$ and $Q^{x}, \widetilde{P}^{x}$ be the processes associated with $\left(x_{t}, \mathscr{F}_{t}, P\right)$ and $\left(x_{t}, \mathscr{F}_{t}^{x}, P\right)$. Similarly let $Q_{1}, \widetilde{P}_{1}, Q_{1}^{x}, \widetilde{P}_{1}^{x}$ be the processes corresponding with $\left(x_{t}, \mathscr{F}_{t}, P_{1}\right)$ and $\left(x_{t}, \mathscr{F}_{t}^{x}, P_{1}\right)$ respectively. From Assumption 5.2 and Proposition 4.1 it is immediate that

$$
\begin{aligned}
\widetilde{P}(B, t) & =\tilde{P}^{x}(B, t)=\mu(B, t), \\
\tilde{P}_{1}(B, t) & =\int_{B} \int_{0}^{t}(1+f(z, s)) \mu(d z, d s), \\
\tilde{P}_{1}^{x}(B, t) & =\int_{B} \int_{0}^{t}(1+\hat{f}(z, s)) \mu(d z, d s),
\end{aligned}
$$

where $\hat{f}(z, t)=E_{1}\left(f(z, t) \mid \mathscr{F}_{t}^{x}\right)$.

For any $t$ let $\mathscr{F}_{t^{-}}=V_{s<t} \mathscr{F}_{s}, \mathscr{F}_{t^{-}}^{x}=\bigvee_{s<t} \mathscr{F}_{s}^{x}, \mathscr{F}_{t^{-}}^{y}=\bigvee_{s<t} \mathscr{F}_{s}^{y}$.

Proposition 5.1. For $t \in R_{+}, \mathscr{F}_{t^{-}}^{x}=\mathscr{F}_{t}^{x}, \mathscr{F}_{t^{-}}^{y}=\mathscr{F}_{t}^{y}, \mathscr{F}_{t^{-}}=\mathscr{F}_{t}$.

Proof. The jump times of $x$ and $y$ are totally inaccessible, hence by [4, Prop. 3.1] and [22, § III, D38], $\mathscr{F}_{t^{-}}^{x}=\mathscr{F}_{t}^{x}$ and $\mathscr{F}_{t^{-}}^{y}=\mathscr{F}_{t}^{y}$. The last assertion follows because $\mathscr{F}_{t^{-}}=\mathscr{F}_{t^{-}}^{x} \vee \mathscr{F}_{t^{-}}^{y}$ and $\mathscr{F}_{t}=\mathscr{F}_{t}^{x} \vee \mathscr{F}_{t}^{y}$.

Proposition 5.2. $L_{t^{-}}=L_{t}$ a.s. $P$.

Proof. The proof follows from [22, $\S \mathrm{V}$, Thm. 10] using a stopping time argument. 
Proposition 5.3. $y_{t}=y_{t}$ - a.s. $P$.

Proof. Prob $\left\{y_{t} \neq y_{t^{-}}\right\}=\operatorname{Prob}\{t$ is a jump time $\}$. However, since the jump times are totally inaccessible, this probability must be zero.

For a real-valued function $g$ on $Y$ we are interested in determining a (recursive) expression for the process $E_{1}\left(g\left(y_{t}\right) \mid \mathscr{F}_{t}^{x}\right)$. Now

$$
E_{1}\left(g\left(y_{t}\right) \mid \mathscr{F}_{t}^{x}\right)=\frac{E\left(g\left(y_{t}\right) L_{t} \mid \mathscr{F}_{t}^{x}\right)}{E\left(L_{t} \mid \mathscr{F}_{t}^{x}\right)}
$$

It turns out that the numerator of the expression in the right is much better behaved than the ratio, and, furthermore, the denominator does not depend on $g$. Hence we will seek to determine instead an expression for $E\left(g\left(y_{t}\right) L_{t} \mid \mathscr{F}_{t}^{x}\right)$.

Definition 5.1. Let $\mathscr{G}$ be the family of all bounded, measurable, real-valued functions $g$ on $Y$. For $g \in \mathscr{G}$ and $t \in R_{+}$, let

$$
\pi_{t}(g)=E\left(g\left(y_{t}\right) L_{t} \mid \mathscr{F}_{t}^{x}\right) .
$$

Proposition 5.4. $E\left(L_{t} \mid \mathscr{F}_{t}^{y}\right)=1$ a.s.

Proof. The proof is immediate from the assumptions that $\mathscr{F}^{x}, \mathscr{F}^{y}$ are independent under $P$ and $\mu(B, t)$ is $\mathscr{F}_{t}^{x}$-measurable.

Now fix $g \in \mathscr{G}$. Since $L_{t}$ satisfies

substitution into (5.10) gives

$$
L_{t}=1+\int_{0}^{t} L_{s^{-}} d(\phi \circ Q)_{s}
$$

$$
\begin{aligned}
\pi_{t}(g) & =E\left(g\left(y_{t}\right) \mid \mathscr{F}_{t}^{x}\right)+E\left[\int_{0}^{t} g\left(y_{t}\right) L_{s^{-}} d(\phi \circ Q)_{s} \mid \mathscr{F}_{t}^{x}\right] \\
& =E\left(g\left(y_{t}\right) \mid \mathscr{F}_{t}^{x}\right)+\int_{Z} \int_{0}^{t} E\left[g\left(y_{t}\right) L_{s^{-}} \phi\left(z, y_{s^{-}}, s\right) \mid \mathscr{F}_{t}^{x}\right] Q(d z, d s) .
\end{aligned}
$$

Since $\mathscr{F}^{x}$ and $\mathscr{F}^{y}$ are independent under $P$,

$$
E\left(g\left(y_{t}\right) \mid \mathscr{F}_{t}^{x}\right)=E g\left(y_{t}\right)
$$

Also,

$$
\begin{aligned}
E\left[g\left(y_{t}\right) L_{s^{-}} \phi\left(z, y_{s^{-}}, s\right) \mid \mathscr{F}_{t}^{x}\right] \\
\quad=E\left[g\left(y_{t}\right) L_{s} \phi\left(z, y_{s}, s\right) \mid \mathscr{F}_{t}^{x}\right] \\
\quad=E\left[E\left\{g\left(y_{t}\right) L_{s} \phi\left(z, y_{s}, s\right) \mid \mathscr{F}_{t}^{x} \vee \mathscr{F}_{s}^{y}\right\} \mid \mathscr{F}_{t}^{x}\right] \\
\quad=E\left[L_{s} \phi\left(z, y_{s}, s\right) E\left(g\left(y_{t}\right) \mid \mathscr{F}_{t}^{x} \vee \mathscr{F}_{s}^{y}\right) \mid \mathscr{F}_{t}^{x}\right] \\
\quad=E\left[L_{s} \phi\left(z, y_{s}, s\right) E\left(g\left(y_{t}\right) \mid \mathscr{F}_{s}^{y}\right) \mid \mathscr{F}_{t}^{x}\right] \\
\quad=E\left[L_{s} \phi\left(z, y_{s}, s\right) E\left(g\left(y_{t}\right) \mid y_{s}\right) \mid \mathscr{F}_{t}^{x}\right] \\
\quad=E\left[L_{s} \phi\left(z, y_{s}, s\right) H_{t, s}(g) \mid \mathscr{F}_{s}^{x}\right]
\end{aligned}
$$

(by independence of $\mathscr{F}^{x}, \mathscr{F}^{y}$ )

(since $y_{t}$ is Markov)

since $x$ has independent increments under $P$ and where

$$
H_{t, s}(g)=E\left(g\left(y_{t}\right) \mid y_{s}\right) \text {. }
$$


Substitution of (5.12) and (5.13) into (5.11) gives

$$
\pi_{t}(g)=E g\left(y_{t}\right)+\int_{Z} \int_{0}^{t} \pi_{s}\left(\phi(z, \cdot, s) H_{t, s}(g)\right) Q(d z, d s) .
$$

Note that the integrand in the above expression is a predictable process for each fixed $t$, as explained at the end of $\S 2.9$.

We summarize the above.

TheOREM 5.2. Under Assumption (5.2) the process $\pi_{t}(g)$ satisfies

$$
\pi_{t}(g)=E g\left(y_{t}\right)+\int_{Z} \int_{0}^{t} \pi_{s}\left(\phi(z, \cdot, s) H_{t, s}(g)\right) Q(d z, d s),
$$

where

$$
H_{t, s}(g)=E\left(g\left(y_{t}\right) \mid y_{s}\right)
$$

and

$$
Q(B, t)=P(B, t)-\tilde{P}(B, t) .
$$

Remark 5.2. (i) Because of Proposition 5.4,

$$
E g\left(y_{t}\right)=E_{1} g\left(y_{t}\right) \text { and } H_{t, s}(g)=E_{1}\left(g\left(y_{t}\right) \mid y_{s}\right) .
$$

(ii) From $(5.10), \pi_{t}(1)=E\left(L_{t} \mid \mathscr{F}_{t}^{x}\right)$, where 1 denotes the function on $Y$ which is identically equal to unity. Hence from (5.9),

$$
\begin{aligned}
E_{1}\left(g\left(y_{t}\right) \mid \mathscr{F}_{t}^{x}\right) & =\frac{\pi_{t}(g)}{\pi_{t}(1)} \\
& =\frac{E g\left(y_{t}\right)+\int_{Z} \int_{0}^{t} \pi_{s}\left(\phi(z, \cdot, s) H_{t, s}(g)\right) Q(d z, d s)}{1+\int_{Z} \int_{0}^{t} \pi_{s}(\phi(z, \cdot, s)) Q(d z, d s)}
\end{aligned}
$$

from (5.14).

(iii) Suppose $\left(x_{t}, \mathscr{F}_{t}, P\right)$ is as in the fundamental example. Then (5.14) simplifies to

$$
\pi_{t}(g)=E g\left(y_{t}\right)+\sum_{i=1}^{n} \int_{0}^{t} \pi_{s}\left(\phi_{i}(\cdot, s) H_{t, s}(g)\right)\left[p_{i}(d s)-\tilde{p_{i}}(d s)\right] .
$$
$A \in \mathscr{Y}$,

(iv) We now derive a more familiar-looking version of (5.14). For any set

$$
\pi_{t}\left(I_{A}\right)=E\left(I_{A} L_{t} \mid \mathscr{F}_{t}^{x}\right) .
$$

If $P\left(y_{t} \in A\right)=P_{1}\left(y_{t} \in A\right)=0$, then $\pi_{t}\left(I_{A}\right)=0$ a.s. Hence there exists a measurable function $U_{t}: Y \rightarrow R$ such that

$$
\pi_{t}(A)=\int_{A} U_{t}(y) P_{t}(d y)
$$

where $P_{t}$ is the marginal distribution of $y_{t}$ under $P$ and $P_{1}$. Evidently if $h \in \mathscr{G}$, then

$$
\pi_{t}(h)=\int_{Y} h(y) U_{t}(y) P_{t}(d y)
$$


Next let $P(A, t \mid y, s), A \in \mathscr{Y}, s \leqq t$, be the transition kernel of the Markov process $y$ so that

$$
\left(H_{t, s}(g)\right)(y)=\int_{Y} g\left(y^{\prime}\right) P\left(d y^{\prime}, t \mid y, s\right)
$$

and let $P(A, s \mid y, t), A \in \mathscr{Y}, t \geqq s$, be the backward kernel so that for $h \in \mathscr{G}$,

$$
E\left(h\left(y_{s}\right) \mid y_{t}\right)=\int_{Y} h\left(y^{\prime}\right) P\left(d y^{\prime}, s \mid y_{t}, t\right) .
$$

Substituting these relations into (5.14) leads to

$$
\begin{aligned}
\int_{Y} g\left(y^{\prime}\right) U_{t}\left(y^{\prime}\right) P_{t}\left(d y^{\prime}\right)= & \int_{Y} g\left(y^{\prime}\right) P_{t}\left(d y^{\prime}\right)+\int_{Z} \int_{0}^{t}\left[\int_{Y}\{\phi(z, y, s)\right. \\
& \left.\left.\cdot \int_{Y} g\left(y^{\prime}\right) P\left(d y^{\prime}, t \mid y, s\right)\right\} U_{s}(y) P_{s}(d y)\right] \cdot Q(d z, d s) \\
= & \int_{Y} g\left(y^{\prime}\right) P_{t}\left(d y^{\prime}\right)+\int_{Y} g\left(y^{\prime}\right) \\
& \cdot\left[\int_{Z} \int_{0}^{t}\left\{\int_{Y} \phi(z, y, s) U_{s}(y) P\left(d y, s \mid y^{\prime}, t\right)\right\} Q(d z, d s)\right] \cdot P_{t}\left(d y^{\prime}\right) .
\end{aligned}
$$

Since $g \in \mathscr{G}$ is arbitrary, the process $U_{t}(y)$ evolves according to

$$
U_{t}(y)=1+\int_{Z} \int_{0}^{t}\left[\int_{Y} \phi\left(z, y^{\prime}, s\right) U_{s}\left(y^{\prime}\right) P\left(d y^{\prime}, s \mid y, t\right)\right] Q(d z, d s) .
$$

Remark 5.3. (i) For the case of the fundamental example (see (5.17)) the equation above simplifies to

$$
U_{t}(y)=1+\sum_{i=1}^{n} \int_{0}^{t}\left\{\int_{Y} \phi_{i}\left(y^{\prime}, s\right) U_{s}\left(y^{\prime}\right) P\left(d y^{\prime}, s \mid y, t\right)\right\}\left[p_{i}(d s)-\tilde{p}_{i}(d s)\right] .
$$

This equation has been derived in [28] for the special case where $\left(x_{t}, \mathscr{F}_{t}, P\right)$ is a counting process, so that $n=1$, and with the additional condition that $\tilde{p}(d s)=d s$.

(ii) Equations (5.14) and (5.15) are not yet recursive since the functions $\phi(z, y, t), \phi_{i}(y, t)$ are allowed to depend on the entire past $x_{s}, 1 \leqq s \leqq t$. We will see later how under additional conditions these equations become truly recursive.

(iii) Notice that unlike the representation for $\hat{y}_{t}$ obtained in Theorem 5.1, those for $\pi_{t}$ in (5.14) and $U_{t}$ in (5.19) are not semimartingales because the integrands depend upon $t$. This dependency can be eliminated by some additional assumptions as follows.

For the remainder of this section the following holds in addition to Assumption 5.2.

Assumption 5.3. The operators $H_{t, s}$ of (5.15) have the following properties:

$$
\lim _{s \uparrow t} H_{t, s}=I \text {, the identity operator on } \mathscr{G} \text {. }
$$


(ii) there exist operators $A_{t}, t \geqq 0$ on $\mathscr{G}$ such that

$$
\lim _{\varepsilon \downarrow 0}(1 / \varepsilon)\left(H_{t+\varepsilon, s}-H_{t, s}\right)(g)=H_{t, s} A_{t}(g) .
$$

We do not elaborate on the precise theoretical status of the operators $A_{t}$ (i.e., the precise definitions of their domain, range, etc.), since it would take us too far afield and since this topic is well-covered in the semigroup theory of Markov processes (see, e.g., [29]). We merely note that (i) is a continuity assumption, (ii) is a differentiability assumption. The operators $A_{t}$ are often referred to as the infinitesimal generator, especially when $y$ is a Hunt process. If $y$ is a $k$-dimensional diffusion, for example, then $A_{t}$ is just a (partial) differential operator of the form

$$
\frac{1}{2} \sum_{i, j=1}^{k} \sigma_{i j}(y, t) \frac{\partial^{2}}{\partial y_{i} \partial y_{j}}+\sum_{i=1}^{k} m_{i}(y, t) \frac{\partial}{\partial y_{i}} .
$$

We now develop the simplifications induced by (5.21), (5.22) in (5.14). First of all, recalling that $P_{0}(d y)$ is the probability distribution of $y_{0}$ and that $y$ is Markov, we get

$$
E g\left(y_{t}\right)=\int_{Y}\left(H_{t, 0}(g)\right)(y) P_{0}(d y)=E\left(H_{t, 0}(g)\left(y_{0}\right)\right)
$$

This, together with (5.22), implies that

$$
\begin{aligned}
E\left(g\left(y_{t+\varepsilon}\right)-g\left(y_{t}\right)\right) & =\int_{Y}\left(H_{t+\varepsilon, 0}-H_{t, 0}\right)(g)(y) P_{0}(d y) \\
& \cong \varepsilon \int_{Y}\left(H_{t, 0} A_{t}(g)\right)(y) P_{0}(d y)=\varepsilon E\left[\left(A_{t}(g)\right)\left(y_{t}\right)\right] .
\end{aligned}
$$

Substituting this into (5.14), and using (5.21) and (5.22), leads us to

$$
\begin{aligned}
\left(\pi_{t+\varepsilon}-\pi_{t}\right)(g) & \cong \varepsilon E\left(A_{t}(g)\right)+\varepsilon \int_{Z} \pi_{t}(\phi g) Q(d z, d t)+\varepsilon \int_{Z} \int_{0}^{t} \pi_{s}\left(\phi H_{t, s} A_{t}(g)\right) Q(d z, d s) \\
& =\varepsilon \int_{Z} \pi_{t}(\phi g) Q(d z, d t)+\varepsilon \pi_{t}\left(A_{t}(g)\right) .
\end{aligned}
$$

Hence

$$
\pi_{t}(g)=\pi_{0}(g)+\int_{0}^{t} \pi_{s}\left(A_{s} g\right) d s+\int_{0}^{t} \int_{Z} \pi_{s}(\phi(z, \cdot, s) g) Q(d z, d s) .
$$

THEOREM 5.3. Under the additional conditions of Assumption 5.3, the representations (5.14) and (5.17) simplify to (5.23), (5.24) respectively.

$$
\pi_{t}(g)=\pi_{0}(g)+\int_{0}^{t} \pi_{s}\left(A_{s} g\right) d s+\sum_{i=1}^{n} \int_{0}^{t} \pi_{s}\left(\phi_{i}(\cdot, s) g\right)\left[p_{i}(d s)-\tilde{p}_{i}(d s)\right] .
$$

As an example illustrating (5.24) suppose that under $P x_{t}$ and $y_{t}$ are independent standard Poisson processes. Then $Z=Y=I_{+}$, the set of nonnegative 
integers. Also $n=1$ in (5.24), $p(t)=x_{t}$ and $\tilde{p}(t)=t$. For $g: I_{+} \rightarrow R$,

$$
\begin{aligned}
H_{t, s}(g)(y) & =E\left(g\left(y_{t}\right) \mid y_{s}=y\right) \\
& =\sum_{k=0}^{\infty} g(y+k) \frac{(t-s)^{k}}{k !} e^{-(t-s)},
\end{aligned}
$$

so that,

$$
\frac{\partial}{\partial t}\left(H_{t, s}(g)\right)(y)=\sum_{k=0}^{\infty} \frac{(t-s)^{k}}{k !} e^{-(t-s)}[g(y+k+1)-g(y+k)],
$$

and hence

$$
\left(A_{t} g\right)(y)=(A g)(y)=g(y+1)-g(y) .
$$

Consider the "indicator" functions $\delta_{k}: I_{+} \rightarrow R$, where

$$
\delta_{k}(y)= \begin{cases}1 & \text { if } y=k \\ 0 & \text { otherwise. }\end{cases}
$$

By the linearity of $\pi_{t}$,

$$
\pi_{t}(g)=\sum_{k=0}^{\infty} g(k) \pi_{t}\left(\delta_{k}\right)
$$

so that it is enough to determine the processes $\pi_{t}\left(\delta_{k}\right), k=0,1,2, \cdots$. Substitution of $\delta_{k}$ for $g$ into (5.24) gives, using (5.25),

$$
\begin{aligned}
\pi_{t}\left(\delta_{k}\right) & =\pi_{0}\left(\delta_{k}\right)+\int_{0}^{t}\left[\pi_{s}\left(\delta_{k-1}\right)-\pi_{s}\left(\delta_{k}\right)\right] d s+\int_{0}^{t} \pi_{s}\left(\phi(\cdot, s) \delta_{k}\right)\left(d x_{s}-d s\right) \\
& =\pi_{0}\left(\delta_{k}\right)+\int_{0}^{t}\left[\pi_{s}\left(\delta_{k-1}\right)-\pi_{s}\left(\delta_{k}\right)\right] d s+\int_{0}^{t} \phi(k, s) \pi_{s}\left(\delta_{k}\right)\left(d x_{s}-d s\right),
\end{aligned}
$$

since $\phi(y, s) \delta_{k}(y)=\phi(k, s) \delta_{k}(y)$. Now

$$
\pi_{0}\left(\delta_{k}\right)=E \delta_{k}\left(y_{0}\right)= \begin{cases}1 & \text { if } k=0 \\ 0 & \text { if } k>0\end{cases}
$$

and $\delta_{-1} \equiv 0$, so that the expression above simplifies to

$$
\begin{aligned}
& \pi_{t}\left(\delta_{0}\right)=1+\int_{0}^{t} \pi_{s}\left(\delta_{0}\right) d s+\int_{0}^{t} \phi(0, s) \pi_{s}\left(\delta_{0}\right)\left(d x_{s}-d s\right), \\
& \pi_{t}\left(\delta_{k}\right)=\int_{0}^{t} \pi_{s}\left(\delta_{k-1}\right) d s-\int_{0}^{t} \pi_{s}\left(\delta_{k}\right) d s+\int_{0}^{t} \phi(k, s) \pi_{s}\left(\delta_{k}\right)\left(d x_{s}-d s\right), \quad k \geqq 1,
\end{aligned}
$$

and these can be rewritten respectively as

$$
\begin{aligned}
& e^{t} \pi_{t}\left(\delta_{0}\right)=1+\int_{0}^{t} \phi(0, s) e^{s} \pi_{s}\left(\delta_{0}\right)\left(d x_{s}-d s\right) \\
& e^{t} \pi_{t}\left(\delta_{k}\right)=\int_{0}^{t} e^{s} \pi_{s}\left(\delta_{k-1}\right) d s+\int_{0}^{t} \phi(k, s) e^{s} \pi_{s}\left(\delta_{k}\right)\left(d x_{s}-d s\right), \quad k \geqq 1 .
\end{aligned}
$$


These linear integral equations can now be solved inductively to yield the explicit formulas

$$
\begin{aligned}
& \pi_{t}\left(\delta_{0}\right)=e^{-t} \prod_{\substack{s \leqq t \\
x_{s}-\neq x_{s}}}[1+\phi(0, s)] \exp \left[-\int_{0}^{t} \phi(0, s) d s\right], \\
& \pi_{t}\left(\delta_{k}\right)=\int_{0}^{t} e^{-(t-s)} \pi_{s}\left(\delta_{k-1}\right)\left\{\prod_{\substack{s<\tau \leqq t \\
x_{\tau^{-}} \neq x_{\tau}}}[1+\phi(k, \tau)] \exp \left[-\int_{s}^{t} \phi(k, \tau) d \tau\right]\right\} d s, \\
& k \geqq 1 .
\end{aligned}
$$

Remark 5.4. The result just obtained illustrates the power of the formulation of Theorem 5.3 over the more usual formulations which involve obtaining a relation for the conditional density (e.g., [16]). We believe that equations (5.14), (5.20), and (5.23), (5.24) are much more useful since they are linear in the "unknown" linear operators $\pi_{t}$ whereas the evolution equations for the conditional density are nonlinear. Of course the latter can be easily derived from the former.

\section{REFERENCES}

[1] C. DolÉANS-DADE AND P. A. MEYER, Intégrales stochastiques par rapport aux martingales locales, Séminaire de Probabilitès: IV, Lecture Notes in Mathematics, Springer-Verlag, Berlin and New York, 1970, pp. 77-107.

[2] C. DolÉANS-DADE, Quelques applications de la formule de changement de variables pour les semimartingales, Z. Wahrscheinlichtstheorie Verw. Gebiete, 16 (1970), pp. 181-194.

[3] P. A. Meyer, Probabilités et Potentiel, Hermann, Paris, 1966-English translation: Probability and Potential, Blaisdell, Waltham, Mass., 1966.

[4] R. Boel, P. VARAIYA AND E. Wong, Martingales on point processes I: Representation results, Memo \# M-407, Electronics Research Lab., University of California, Berkeley, Calif., 1973.

[5] H. Kunita AND S. Watanabe, On square integrable martingales, Nagoya Math. J., 30 (1967), pp. 209-245.

[6] P. M. BRÉmaud, A martingale approach to point processes, Electronics Research Lab., Memo \# M-345, University of California, Berkeley, Calif., 1972.

[7] M. H. A. Davis, Detection of signals with point process observation, Publication 73/8, Dept. of Computing and Control, Imperial College, London, 1973.

[8] T. T. KAdota AND L. A. SHEPP, Conditions for absolute continuity between a certain pair of probability measures, Z. Wahrscheinlichtstheorie Verw. Gebiete (3), 16 (1960), pp. 13-30.

[9] I. V. GiRSANOv, On transforming a certain class of stochastic processes by absolutely continuous substitution of measures, Theory Probability Appl., 5 (1960), pp. 285-301.

[10] V. BENES, Existence of optimal stochastic control laws, this Journal, 9 (1971), pp. 446475.

[11] T. E. Duncan AND P. VARAIYA, On the solutions of a stochastic control system, this Journal, 9 (1971), pp. 354-371.

[12] M. H. A. Davis AND P. VARAIYA, Dynamic programming conditions for partially observable stochastic systems, this Journal, 11 (1973), pp. 226-261.

[13] R. RisHeL, Weak solutions of a partial differential equation of dynamic programming, this Journal, 9 (1971), pp. 519-528.

[14] D. L. SNYDER, Information processing for observed jump processes, Information and Control, 22 (1973), pp. 69-78.

[15] I. RUBIN, Regular point processes and their detection, IEEE Trans. Information Theory IT-18 (1972), pp. 547-557.

[16] D. L. SNYDER, Filtering and detection for doubly stochastic Poisson processes, Ibid., IT-18 (1972), pp. 91-102.

[17] O. MaCChi AND B. C. Picinbono, Estimation and detection of weak optical signals, Ibid., IT-18 (1972), pp. 562-573. 
[18] E. Lehmann, Testing Statistical Hypotheses, Wiley, New York, 1959.

[19] P. A. MEYER, Square integrable martingales, a survey, Martingales: A report on a Meeting at Oberwolfach, Lecture Notes in Mathematics, No. 190, Springer-Verlag, Berlin, 1970.

[20] M. H. A. DAvIs, Nonlinear filtering with point process observations, preprint.

[21] M. Fujisaki, G. KallianPUR AND H. Kunita, Stochastic differential equations for the nonlinear filtering problem, Osaka J. Math. (1), 9 (1972), pp. 19-40.

[22] C. Dellacherie, Capacités et processus stochastiques, Springer-Verlag, Berlin, 1972.

[23] T. E. Duncan, On the absolute continuity of measures, Annals Math. Statist., 41 (1970), pp. 30-38.

[24] J. H. VAN SCHUPPEN, Estimation theory for continuous time processes, a martingale approach, Memo \# M-405, Electronics Research Lab., University of California, Berkeley, Calif., 1973.

[25] J. L. Doob, Stochastic Processes, Wiley, New York, 1953.

[26] W. Feller, An Introduction to Probability Theory and Its Applications, v.I, Wiley, New York, 1968.

[27] T. E. Duncan and P. Varaiya, On the solutions of a stochastic control system II, Memo \# M406, Electronics Research Lab., Univ. of California, Berkeley, Calif., 1973.

[28] P. BRÉMAUD, Filtering for point processes, preprint, 1973.

[29] E. B. Dynkin, Markov Processes I, Academic Press, New York, 1965.

[30] D. L. SNYDER, Smoothing for doubly stochastic Poisson processes, IEEE Trans. Information Theory, IT-18 (1972), pp. 558-562.

[31] A. Prékopa, On stochastic set functions, Acta Math. Acad. Sci. Hungar. (2), 7 (1956), pp. 215-263.

[32] J. E. MoYAL, The general theory of stochastic population processes, Acta Math. (Uppsala), 108 (1962), pp. 1-31.

[33] J. H. VAN SchuPPEN, Filtering for counting processes, a martingale approach, Proc. 4th Symp. Nonlinear Estimation and Appl., San Diego, Calif., 1973. 